\title{
Semi-Infinite Homology and 2D Gravity. I
}

\author{
Bong H. Lian ${ }^{1,2, \star}$ and Gregg J. Zuckerman ${ }^{3, \star \star}$ \\ 1 Yale University, Center for Theoretical Physics, New Haven, CT 06520, USA \\ 2 University of Toronto, Department of Mathematics, Toronto, Ontario, Canada M5S 1A1 \\ 3 Yale University, Department of Mathematics, New Haven, CT 06520, USA
}

Received August 2, 1991; in revised form November 15, 1991

\begin{abstract}
In [33], we studied the constraint problem for two-dimensional quantum gravity in the conformal gauge. In this gauge, we proposed an ansatz for the gravitational sector. Using this ansatz, we established a striking connection between the matrix models and continuum 2D gravity. We also announced several results on semi-infinite homology of the Virasoro algebra with coefficients in a suitable class of positive energy modules. In this article, we will provide details of the proof of the announced results.
\end{abstract}

\section{Introduction}

\subsection{Motivation}

The Liouville theory has been the focus of a number of recent discussions in the contexts of two-dimensional quantum gravity and non-critical strings [14, 35, 42, $11,10]$. When $c<1$ matter is coupled to $2 \mathrm{D}$ gravity, certain aspects of the theory critical exponents, string susceptibilities, correlation functions - have been extensively studied via both the continuum and matrix-model approaches. There is, however, one fundamental aspect of the theory which lacks the same level of understanding - what are the basic building blocks of the physical state space?

If we consider 2D gravity coupled to a conformal field theory (matter) in the conformal gauge, then gauge fixing results in the Virasoro constraint [11]

$$
T^{\mathrm{CFT}}(z)+T^{\varphi}(z)+T^{b c}(z) \sim 0,
$$

where $T^{\mathrm{CFT}}(z), T^{\varphi}(z), T^{b c}(z)$, are the stress-energy tensors of the CFT, the Liouville field $\varphi$, and the conformal ghosts respectively. $T^{\varphi}(z)$ has the form $[11,42]$

$$
T^{\varphi}(z)=-\frac{1}{2}(\partial \varphi)^{2}+i \xi \partial^{2} \varphi
$$

\footnotetext{
* Supported in part by the Alfred P. Sloan Foundation

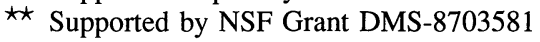


where $\xi$ is a parameter of the theory. Since (1.1) is a first class constraint, a natural way to quantize the theory is the BRST procedure. Thus it is clear that the basic building blocks of the physical state space, in the presence of a matter CFT, should be the BRST homology classes. These homology classes depend on the choice of the matter and on the representation of the Virasoro algebra in the Liouville sector. We will begin with the minimal models as matter. A natural class of representations in the Liouville sector is given by the free field realizations of the Virasoro algebra [8, 9, 22], also known as the Feigin-Fuchs modules. The importance of these modules has been demonstrated both in physics (see for example [20]) and in mathematics [18]. In our previous announcement [33], we determined exactly which of these modules lead to non-trivial BRST homology. In this article, we provide details of the proof. Our ansatz for the gravitational sector has also been used for studying $c=1$ boson coupled to gravity [34]. The $c=1$ theory was shown to be substantially different from the $c<1$ theory.

We begin by briefly reviewing the BRST formalism. In addition to the FeiginFuchs modules, two other classes of Vir representations - the Verma modules and their irreducible quotients - arise naturally in our discussion. In fact in our earlier announcement, some key results concerning all three classes of modules were stated (Theorems 1-3 of [33]). Here, we will devote Sects. 2 and 3 to the proofs of these results. We then conclude with a few remarks and some preliminary results on supergravity. More detailed discussions can be found in [31].

We thank A. Rocha for helpful discussion. We especially thank C. Crnkovic and G. Moore for numerous valuable discussions during the course of this work.

\subsection{The BRST Formalism}

For a mathematical review of the BRST theory of graded Lie algebras, see [17, 21, $32,31]$. The content of $2 \mathrm{D}$ gravity coupled to matter in the conformal gauge has the form

Liouville $\otimes$ Matter $\otimes$ Ghosts .

This system has a first class constraint given by the algebra Vir $\oplus \overline{\operatorname{Vir}}$. For simplicity, we will focus, throughout this article, on the left-handed part of theory.

Upon quantization, the Liouville sector is represented by the Feigin-Fuchs modules $F_{\xi, \eta}$. They are the free-field representations in which the Virasoro algebra, Vir, acts by:

$$
T^{L}(z)=\frac{1}{2}: j(z)^{2}:+\xi \partial j(z)
$$

with central charge

$$
c_{L}=1-12 \xi^{2}
$$

The space $F_{\xi, \eta}$ is the linear span of the vectors

$$
j_{n_{1}} j_{n_{2}} \ldots j_{n_{k}} v_{\xi, \eta} \quad n_{1} \leq n_{2} \leq \ldots n_{k}<0 .
$$

The modes of $j(z)$ satisfy

$$
\begin{aligned}
{\left[j_{n}, j_{m}\right] } & =n \delta_{n+m, 0}, \\
j_{n} v_{\xi, \eta} & =(\xi-\eta) \delta_{n, 0} v_{\xi, \eta} \quad n \geq 0 .
\end{aligned}
$$

A natural class of matter to be coupled to gravity consists of the conformal field theories (CFT). Associated with each CFT is a collection of modules $M$ over Vir. 
Thus there is a stress-energy $T^{M}(z)$ which operates on each $M$, with central charge $c_{M}$. We will primarily focus on the case when $M$ is a minimal model representation [3]:

$$
\begin{gathered}
M=L\left(c_{p, q}, \Delta_{r, s}\right) \\
c_{M}=c_{p, q}=1-\frac{6(p-q)^{2}}{p q} \\
\Delta_{r, s}=\frac{(p r-q s)^{2}-(p-q)^{2}}{4 p q},
\end{gathered}
$$

where $p, q, r, s$ are positive integers with $\operatorname{gcd}(p, q)=1, p r>q s, q>r, p>s$.

The Liouville sector and matter together do not constitute the full quantum state space; neither do they necessarily contain all the physical states. Gauge-fixing of 2D gravity naturally leads to the ghost sector and the BRST constraint, by which physical states are determined.

According to the prescription of BRST quantization, there is, associated with the constraint algebra Vir, a Clifford algebra $\mathscr{C}$ defined by the relations:

$$
\left\{\varepsilon\left(x^{\prime}\right), \iota(y)\right\}=\left\langle x^{\prime}, y\right\rangle \quad x^{\prime} \in \operatorname{Vir}^{\prime}, y \in \text { Vir . }
$$

There is also a representation of $\operatorname{Vir},\left\{\wedge_{\frac{\infty}{2}+*}(\operatorname{Vir} / \mathrm{C} \hat{c}), \eta\right\}$, given by

$$
\eta(x)=\sum_{m}: \varepsilon\left(L_{m}^{\prime}\right) \iota\left(\left[L_{m}, x\right]\right):+\langle\bar{\beta}, x\rangle
$$

with $\bar{\beta}=-L_{0}^{\prime}-26 \hat{c}^{\prime}$. In more familiar notations, we write

$$
\begin{gathered}
c_{g h}=-26, \quad b_{n}=\iota\left(L_{n}\right), \quad c_{n}=\varepsilon\left(L_{-n}^{\prime}\right) \\
L_{n}^{g h}=\eta\left(L_{n}\right)=\sum(m-n): c_{-m} b_{m+n}:-\delta_{n}, \\
T^{g h}(z)=\sum L_{n}^{g h} z^{-n-2}=: c(z) \partial b(z)+2 \partial c(z) b(z): \\
c(z)=\sum c_{n} z^{-n+1}, \quad b(z)=\sum b_{n} z^{-n-2}
\end{gathered}
$$

$c(z), b(z)$ are respectively the ghost and anti-ghost fields. The spaces $\wedge_{\frac{\infty}{2}+*}(\operatorname{Vir} / \mathbf{C} \hat{c})$ in which the ghosts act are spanned by vectors of the form

$$
\begin{gathered}
b_{n_{1}} \ldots b_{n_{i}} c_{m_{1}} \ldots c_{m_{\jmath}} w_{0}, \\
n_{1}<\cdots<n_{i}<0, \quad m_{1}<\cdots<m_{j} \leq 0,
\end{gathered}
$$

where $w_{0}$ is the vacuum vector carrying ghost number zero.

Now the full quantum state space should be the $\frac{\infty}{2}$-chain complex:

$$
C_{\frac{\infty}{2}+*}\left(\operatorname{Vir}, \mathbf{C} \hat{c} ; M \otimes F_{\xi, \eta}\right)=M \otimes F_{\xi, \eta} \otimes \wedge_{\frac{\infty}{2}+*}(\operatorname{Vir} / \mathbf{C} \hat{c}) .
$$

The full gauge constraint to be imposed is

$$
T^{M}(z)+T^{L}(z)+T^{g h}(z) \sim 0 .
$$

In order to get any state to obey (1.19), we must at least have zero total central charge:

$$
c_{M}+c_{L}+c_{g h}=0 \text {. }
$$


The BRST operator is given by

$$
d=\sum\left(L_{n}^{L}+L_{n}^{M}\right) c_{-n}-\frac{1}{2} \sum(n-m): c_{-n} c_{-m} b_{n+m}:-c_{0} .
$$

It can be written as

$$
\begin{gathered}
d=\frac{1}{2 \pi i} \oint J(z) d z \\
J(z)=:\left(T^{M}(z)+T^{L}(z)-b(z) \partial c(z)\right) c(z): .
\end{gathered}
$$

The energy operator in each sector is given by the zeroth component of its stressenergy field. Thus the total energy operator is

$$
L_{0}=L_{0}^{L}+L_{0}^{M}+L_{0}^{g h} .
$$

This operator is BRST exact:

$$
\left\{d, b_{0}\right\}=L_{0} .
$$

Therefore, the subspace annihilated simultaneously by $b_{0}$ and $L_{0}$ is stabilized by $d$. This subspace is the $\frac{\infty}{2}$-complex relative to $\operatorname{Vir}_{0}=\mathbf{C} L_{0}+\mathbf{C} \hat{c}$ :

$$
\begin{aligned}
& C_{\frac{\infty}{2}+*}\left(\operatorname{Vir}, \operatorname{Vir}_{0} ; M \otimes F_{\xi, \eta}\right) \\
& \quad=\left\{\omega \in M \otimes F_{\xi, \eta} \otimes \wedge_{\frac{\infty}{2}+*}(\operatorname{Vir} / \mathbf{C} \hat{c}) \mid L_{0} \omega=0=b_{0} \omega\right\} .
\end{aligned}
$$

This is the main object of our study in this article. We will return to the complex (1.18) later. The homology groups, $H_{\frac{\infty}{2}+*}\left(\operatorname{Vir}, \operatorname{Vir}_{0} ; L\left(c_{p, q}, \Delta_{r, s}\right) \otimes F_{\xi, \eta}\right)$, of this complex are called the semi-infinite homology groups relative to $\operatorname{Vir}_{0}$.

Problem 1. Given $p, q, r, s$, classify the Feigin-Fuchs modules $F_{\xi, \eta}$ for which

$$
H_{\frac{\infty}{2}+*}\left(\operatorname{Vir}, \operatorname{Vir}_{0} ; L\left(c_{p, q}, \Delta_{r, s}\right) \otimes F_{\xi, \eta}\right) \neq 0 \text {. }
$$

This is the equivalent to determining all free field representations of the gravitational sector which result in non-trivial BRST invariant states, in the presence of conformal matter $L\left(c_{p, q}, \Delta_{r, s}\right)$.

Theorem 3.1 (Theorem 3(a) [33]). For $\eta \in \mathbf{C}, H_{\frac{\infty}{2}}\left(\operatorname{Vir}, \operatorname{Vir}_{0} ; L\left(c_{p, q}, \Delta_{r, s}\right) \otimes F_{\xi, \eta}\right) \neq 0$ iff

$$
\eta=\sqrt{-1} \varepsilon_{1} \frac{\left(2 p q t+p r+\varepsilon_{2} q s\right)}{\sqrt{2 p q}}
$$

for some $t \in \mathbf{Z}, \varepsilon_{1}, \varepsilon_{2}= \pm 1$.

If we interpret the zero mode $j_{0}$ as the center-of-mass momentum variable, as one does in the free bosonic string theory, then Problem 1 really amounts to diagonalizing the momentum operator $j_{0}$ in the space of physical states. Thus it is reasonable to ask for the multiplicities of the eigenvalues.

Problem 2. Determine the exact number of BRST invariant states in each case, i.e. calculate $\operatorname{dim} H_{\frac{\infty}{2}+*}\left(\operatorname{Vir}, \operatorname{Vir}_{0} ; L\left(c_{p, q}, \Delta_{r, s}\right) \otimes F_{\xi, \eta}\right)$. Theorem 3.3 (Theorem 3(b) [33]). For $\sqrt{-1} \xi=\frac{p+q}{\sqrt{2 p q}}, \sqrt{-1} \eta=\varepsilon_{1} \frac{2 p q t+p r+\varepsilon_{2} q s}{\sqrt{2 p q}}$
$t \in \mathbf{Z}, \varepsilon_{1}, \varepsilon_{2}= \pm 1$

$$
H_{\frac{\infty}{2}+n}\left(\operatorname{Vir}, \operatorname{Vir}_{0} ; L\left(c_{p, q}, \Delta_{r, s}\right) \otimes F_{\xi, \eta}\right) \cong \delta_{n+\operatorname{sign}(t) d_{\eta}, 0} \mathbf{C},
$$

where $d_{\eta}$ is given by $E q$. (3.55). 
The next two chapters will be devoted to proving the two theorems above. Several examples of the results are illustrated in Figs. 1-4 at the end of Sect. 3.2.

\section{Irreducible and Verma Modules}

Our first step toward solving Problem 1 is to first classify the highest weight irreducible modules $L(c, \Delta)$ which afford non-trivial homology:

$$
H_{\frac{\infty}{2}+*}\left(\operatorname{Vir}, \operatorname{Vir}_{0} ; L\left(c_{p, q}, \Delta_{r, s}\right) \otimes L(c, \Delta)\right) \neq 0 .
$$

The next few sections are devoted to this question. We will return to Problem 1 in the next section. The problem of characterizing (2.1) involves the knowledge of the structure of Verma modules over the Virasoro algebra [18, 19, 28, 39].

\subsection{Structure of Verma Modules}

Recall that for $c, \Delta \in \mathbf{C}$, the Verma module $M(c, \Delta)$ is the largest highest weight module with highest weight $(c, \Delta)$. Given another $M\left(c^{\prime}, \Delta^{\prime}\right)$, we can ask whether there is a non-trivial module map

$$
M\left(c^{\prime}, \Delta^{\prime}\right) \rightarrow M(c, \Delta) .
$$

Note that if it exists, this map is an embedding. Obviously, the map exists only if $c=c^{\prime}$. Since $M\left(c, \Delta^{\prime}\right)$ is generated by a single highest weight vector $v_{\Delta^{\prime}}$ (we suppress $c$ from the notation, and assume that $c$ is fixed) the map (2.2) is clearly determined by the image of $v_{\Delta^{\prime}}$. Because $v_{\Delta^{\prime}}$ is singular

$$
L_{n} v_{\Delta^{\prime}}=0, \quad n>0,
$$

so is its image under (2.2). Thus, that the map (2.2) exists implies that $M(c, \Delta)$ has a singular vector of weight $\Delta^{\prime}$ (we call $\Delta^{\prime}$ a singular weight of $M(c, \Delta)$ ). Conversely, if $M(c, \Delta)$ has a singular vector $u$ of weight $\Delta^{\prime}$, then $U$ (Vir) $\cdot u$ is a highest weight submodule in $M(c, \Delta)$. By universality of $M(c, \Delta)$, there is a unique (injective) map

$$
M\left(c, \Delta^{\prime}\right) \rightarrow M(c, \Delta)
$$

with $v_{\Delta^{\prime}} \mapsto u$. Thus the knowledge of the embeddings among Verma modules is equivalent to knowing the singular weights in every Verma module. If $\Delta^{\prime}$ is a singular weight of $M(c, \Delta)$, we write

$$
\Delta \rightarrow \Delta^{\prime}(\text { at } c)
$$

Thus (2.4), (2.5) are two equivalent statements. We will also use

$$
\Delta \stackrel{\neq}{\rightarrow} \Delta^{\prime}
$$

to mean that $\Delta \rightarrow \Delta^{\prime}$ but $\Delta \neq \Delta^{\prime}$ (again at a given $c$ ).

Theorem 2.1 (Feigin-Fuchs). Every submodule of $M(c, \Delta)$ is a sum of Verma modules. 
Theorem 2.2 (Feigin-Fuchs). The following are the only possible diagrams of the embeddings among Verma modules:

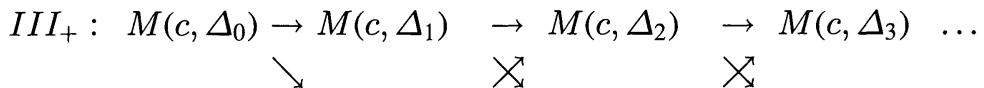

$$
\begin{aligned}
& M\left(c, \Delta_{-1}\right) \rightarrow M\left(c, \Delta_{-2}\right) \rightarrow M\left(c, \Delta_{-3}\right) \ldots \\
& I I I_{-}: M\left(c, \Delta_{0}\right) \leftarrow M\left(c, \Delta_{1}\right) \leftarrow M\left(c, \Delta_{2}\right) \leftarrow M\left(c, \Delta_{3}\right) \ldots
\end{aligned}
$$

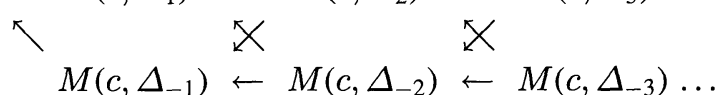

$$
\begin{aligned}
& I I I_{+}^{\prime}: M\left(c, \Delta_{0}\right) \rightarrow M\left(c, \Delta_{1}\right) \rightarrow M\left(c, \Delta_{2}\right) \rightarrow M\left(c, \Delta_{3}\right) \ldots \\
& I I I_{-}^{\prime}: M\left(c, \Delta_{0}\right) \leftarrow M\left(c, \Delta_{1}\right) \leftarrow M\left(c, \Delta_{2}\right) \leftarrow M\left(c, \Delta_{3}\right) \ldots \\
& I I: M\left(c, \Delta_{0}\right) \leftarrow M\left(c, \Delta_{1}\right) \\
& I: M\left(c, \Delta_{0}\right)
\end{aligned}
$$

Each arrow represents a unique map (up to multiple). Every Verma module $M(c, \Delta)$ belongs to a single such diagram. The diagram containing $M(c, \Delta)$ also contains all the Verma modules related to $M(c, \Delta)$ by non-zero maps.

As mentioned earlier, an equivalent description of a map between two Verma modules is an arrow relating their highest weights. The following terminology will be handy later. If $\Delta \rightarrow \Delta^{\prime}\left(\Delta \stackrel{\neq}{\rightarrow} \Delta^{\prime}\right)$, we call $\Delta^{\prime}$ a (proper) descendent of $\Delta ; \Delta$ an (proper) ancestor of $\Delta^{\prime}$. If $\Delta \stackrel{\neq}{\rightarrow} \Delta^{\prime}$ and there is no $\Delta^{\prime \prime}$ such that $\Delta \stackrel{\neq}{\rightarrow} \Delta^{\prime \prime} \stackrel{\neq}{\rightarrow} \Delta^{\prime}$, we call $\Delta^{\prime}$ an immediate descendent of $\Delta ; \Delta$ an immediate ancestor of $\Delta^{\prime}$. If $\Delta \rightarrow \Delta^{\prime}$, we define the distance between them

$$
d\left(\Delta, \Delta^{\prime}\right)=d\left(\Delta^{\prime}, \Delta\right)=\text { max length of chain } \Delta \stackrel{\neq}{\rightarrow} \Delta_{1} \stackrel{\neq}{\rightarrow} \ldots \stackrel{\neq}{\rightarrow} \Delta_{n} \stackrel{\neq}{\rightarrow} \Delta^{\prime} .
$$

Thus $d(\Delta, \Delta)=0$ for any $\Delta ; d\left(\Delta, \Delta^{\prime}\right)=1$ if $\Delta^{\prime}$ is an immediate descendent of $\Delta$.

Feigin and Fuchs proved the two theorems above by studying the geometry of the roots of the Kac determinant formula [28, 18]. Moreover, they gave an algorithm for computing all the ancestors and the descendents of any given $\Delta$. For example, in the $(p, q)$ minimal models, we have

$$
c=c_{p, q}, \quad \Delta=\Delta_{r, s} .
$$

The diagram of $\Delta_{r, s}$ is of type $I I I_{-}$[39]:

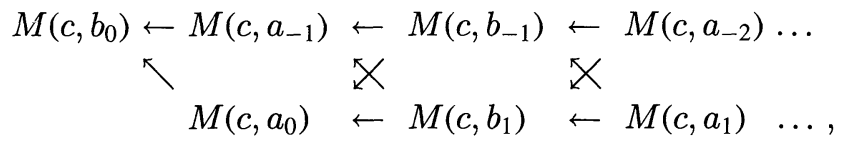

where

$$
\begin{aligned}
& a_{t}=\frac{(2 p q t+p r+q s)^{2}-(p-q)^{2}}{4 p q} \\
& b_{t}=\frac{(2 p q t+p r-q s)^{2}-(p-q)^{2}}{4 p q}
\end{aligned}
$$

for $t \in \mathbf{Z}$.

The following is consequence of the theorems of Feigin-Fuchs. 
Lemma 2.3. $\operatorname{ch} M(c, \Delta)=\sum_{\Delta \rightarrow \Delta^{\prime}} \operatorname{ch} L\left(c, \Delta^{\prime}\right)$, i.e.

$$
\left[M(c, \Delta): L\left(c, \Delta^{\prime}\right)\right]= \begin{cases}1 & \text { if } \Delta \rightarrow \Delta^{\prime} \\ 0 & \text { otherwise }\end{cases}
$$

Feigin and Fuchs discovered yet another remarkable fact about Verma modules. It turns out that

$$
M\left(c, \Delta^{\prime}\right) \hookrightarrow M(c, \Delta) \quad \text { iff } \quad M\left(26-c, 1-\Delta^{\prime}\right) \hookleftarrow M(26-c, 1-\Delta)
$$

("reflection principle"). Thus given a diagram $D$ in Theorem 2.2, the new diagram $\tilde{D}$, obtained by replacing every $M(c, \Delta)$ in $D$ by $M(26-c, 1-\Delta)$ and reversing the arrows in $D$, is also an embedding diagram. We call $\tilde{D}$ the reflection image of $D$. This will be a useful way to get new embedding diagrams.

\subsubsection{Structure of Irreducible Highest Weight Modules}

Proposition 2.4. Every irreducible $L(c, \Delta)$ has a resolution by Verma modules. In particular, there is an exact sequence

$$
\cdots \rightarrow M_{2} \rightarrow M_{1} \rightarrow M_{0} \rightarrow L(c, \Delta) \rightarrow 0
$$

where $M_{i}$ is either zero or a direct sum of Verma modules $M\left(c, \Delta^{\prime}\right)$ with $d\left(\Delta, \Delta^{\prime}\right)=i$.

Proof. The special cases $c=0,1,25,26$ were considered by Rocha-Wallach [41], and $c=c_{p, q}, \Delta=\Delta_{r, s}$ was considered by Feigin-Fuchs [19] and Rocha [39]. For later reference, we will give a uniform treatment here.

Case 1. $\Delta$ has no proper descendent. This means that $M(c, \Delta)$ is irreducible. So

$$
0 \rightarrow M(c, \Delta) \stackrel{\text { id }}{\rightarrow} L(c, \Delta) \rightarrow 0
$$

is exact.

Case 2. $\Delta$ has a single immediate descendent $M\left(c, \Delta^{\prime}\right)$. This means that $M\left(c, \Delta^{\prime}\right)$ is the maximal submodule of $M(c, \Delta)$. Thus

$$
0 \rightarrow M\left(c, \Delta^{\prime}\right) \hookrightarrow M(c, \Delta) \rightarrow M(c, \Delta) / M\left(c, \Delta^{\prime}\right)=L(c, \Delta) \rightarrow 0
$$

is exact.

Case 3. $\Delta$ has two immediate descendents $\Delta_{1}, \Delta_{1}^{\prime}$.

Step 1. Let

$$
\begin{aligned}
& M_{0}=M(c, \Delta) \\
& M_{1}=M\left(c, \Delta_{1}\right) \oplus M\left(c, \Delta_{1}^{\prime}\right)
\end{aligned}
$$

Define $f_{1}: M_{1} \rightarrow M_{0}$ by

$$
f_{1}\left(x, x^{\prime}\right)=x-x^{\prime}
$$

for $x \in M\left(c, \Delta_{1}\right), x^{\prime} \in M\left(c, \Delta_{1}^{\prime}\right)$. Then $\operatorname{Im} f_{1}=M\left(c, \Delta_{1}\right)+M\left(c, \Delta_{1}^{\prime}\right)$ is the maximal submodule of $M_{0}$. So

$$
M_{1} \stackrel{f_{1}}{\rightarrow} M_{0} \rightarrow L(c, \Delta) \rightarrow 0
$$

is exact with $\operatorname{Ker} f_{1}=\left\{(x, x) \mid x \in M\left(c, \Delta_{1}\right) \cap M\left(c, \Delta_{1}^{\prime}\right)\right\}$. 
Step 2. We now proceed inductively. According to Theorem 2.2, there are now two possibilities: either $\Delta_{1}, \Delta_{1}^{\prime}$ have a single common immediate descendent $\Delta_{2}$ in which case we let $f_{2}: M_{2} \rightarrow M_{1}$ be as follows:

$$
\begin{gathered}
M_{2}=M\left(c, \Delta_{2}\right), \\
f_{2}(x)=(x, x), \quad x \in M_{2} .
\end{gathered}
$$

Clearly, $f_{2}$ is injective. Since $M\left(c, \Delta_{2}\right)$ is the maximal submodule of $M\left(c, \Delta_{1}\right)$ and $M\left(c, \Delta_{1}^{\prime}\right)$, we have $M\left(c, \Delta_{2}\right)=M\left(c, \Delta_{1}\right)+M\left(c, \Delta_{1}^{\prime}\right)$. Thus

$$
0 \rightarrow M_{2} \stackrel{f_{2}}{\rightarrow} M_{1} \stackrel{f_{1}}{\rightarrow} M_{0} \rightarrow L(c, \Delta) \rightarrow 0
$$

is exact, and we are done; or, $\Delta_{1}, \Delta_{1}^{\prime}$ have two common immediate descendents $\Delta_{2}$, $\Delta_{2}^{\prime}$, in which case we let $f_{2}: M_{2} \rightarrow M_{1}$ be as follows:

$$
\begin{gathered}
M_{2}=M\left(c, \Delta_{2}\right) \oplus M\left(c, \Delta_{2}^{\prime}\right), \\
f_{2}\left(x, x^{\prime}\right)=\left(x-x^{\prime}, x-x^{\prime}\right) .
\end{gathered}
$$

Clearly, $\operatorname{Im} f_{2}=\left\{(y, y) \mid y \in M\left(c, \Delta_{2}\right)+M\left(c, \Delta_{2}^{\prime}\right)\right\}$. Since $M\left(c, \Delta_{2}\right)+M\left(c, \Delta_{2}^{\prime}\right)$ is the maximal submodule of $M\left(c, \Delta_{1}\right)$ and $M\left(c, \Delta_{1}^{\prime}\right)$, we have $M\left(c, \Delta_{2}\right)+M\left(c, \Delta_{2}^{\prime}\right)=$ $M\left(c, \Delta_{1}\right) \cap M\left(c, \Delta_{1}^{\prime}\right)$. Thus

$$
M_{2} \stackrel{f_{2}}{\rightarrow} M_{1} \stackrel{f_{1}}{\rightarrow} M_{0} \rightarrow L(c, \Delta) \rightarrow 0
$$

is exact with $\operatorname{Ker} f_{2}=\left\{(x, x) \mid x \in M\left(c, \Delta_{2}\right) \cap M\left(c, \Delta_{2}^{\prime}\right)\right\}$. We can repeat Step 2 all over again (with $\left(\Delta_{2}, \Delta_{2}^{\prime}\right)$ playing the role of $\left(\Delta_{1}, \Delta_{1}^{\prime}\right)$ etc.). Continuing this way, if the process terminates, we get a finite exact sequence:

$$
0 \rightarrow M_{i} \rightarrow \cdots \rightarrow M_{0} \rightarrow L(c, \Delta) \rightarrow 0
$$

Otherwise, we get an infinite exact sequence

$$
\cdots \rightarrow M_{i} \rightarrow \cdots \rightarrow M_{0} \rightarrow L(c, \Delta) \rightarrow 0 .
$$

In any case, $L(c, \Delta)$ has a resolution.

Corollary 2.5. The formal character of $L(c, \Delta)$ in each case is given as follows:

(a) In Cases 1 and 3 above,

$$
\operatorname{ch} L(c, \Delta) \stackrel{\text { def }}{=} \operatorname{tr}_{L(c, \Delta)} q^{L_{0}-c / 24}=q^{-c / 24} \prod_{n>0}\left(1-q^{n}\right)^{-1} \sum_{\Delta \rightarrow \Delta^{\prime}}(-1)^{d\left(\Delta, \Delta^{\prime}\right)} q^{\Delta^{\prime}} .
$$

(b) In Case 2 above,

$$
\operatorname{ch} L(c, \Delta)=\operatorname{ch} M(c, \Delta)-\operatorname{ch} M\left(c, \Delta^{\prime}\right) .
$$

When one has knowledge of the irreducible characters, there is a useful tool for detecting non-trivial $\frac{\infty}{2}$-homology - namely, the Euler characteristic. Let $V$ be a positive energy Vir-module. Define

$$
\operatorname{Eul}(V)=\sum_{n \in \mathbf{Z}}(-1)^{n} \operatorname{dim} H_{\frac{\infty}{2}+n}\left(\operatorname{Vir}, \operatorname{Vir}_{0} ; L\left(c_{p, q}, \Delta_{r, s}\right) \otimes V\right) .
$$

By the Euler-Poincaré principle,

$$
\operatorname{Eul}(V)=\sum_{n \in \mathbf{Z}}(-1)^{n} \operatorname{dim} C_{\frac{\infty}{2}+n}\left(\operatorname{Vir}, \operatorname{Vir}_{0} ; L\left(c_{p, q}, \Delta_{r, s}\right) \otimes V\right) .
$$


Lemma 2.6. For any positive energy Vir-module $V$, we have

$$
\begin{aligned}
\operatorname{Eul}(V)= & \sum_{s \in \mathbf{Z}}\left[V: L\left(26-c_{p, q}, 1-b_{s}\right)\right]\left(\# \text { descendents of } 1-b_{s}\right) \\
& -\sum_{s \in \mathbf{Z}}\left[V: L\left(26-c_{p, q}, 1-a_{s}\right)\right]\left(\# \text { descendents of } 1-a_{s}\right) .
\end{aligned}
$$

Proof. It is enough to restrict to $V$ in which $\hat{c}$ acts by the scalar $c=26-c_{p, q}$. Let $U$ be the ghost number operator. By Eq. (2.28),

$$
\begin{aligned}
& \operatorname{Eul}(V)=\sum_{n}(-1)^{n} \operatorname{dim}\left[\wedge_{\frac{\infty}{2}+n}\left(\operatorname{Vir} / \operatorname{Vir}_{0}\right) \otimes L\left(c_{p, q}, \Delta_{r, s}\right) \otimes V\right]^{L_{0}} \\
& =\left[\operatorname{tr}_{\wedge \frac{\infty}{2}\left(\mathrm{Vir}_{\mathrm{Vir}}\right)}(-1)^{U} q^{L_{0}} \times \operatorname{tr}_{L\left(c_{p, q}, \Delta_{r, s}\right)} q^{L_{0}} \times \operatorname{tr}_{V} q^{L_{0}}\right] \\
& =\left[q^{-1} \prod_{n>0}\left(1-q^{n}\right)^{2} \times \prod_{n>0}\left(1-q^{n}\right)^{-1} \sum_{\Delta_{r, s} \rightarrow \Delta^{\prime \prime}}(-1)^{d\left(\Delta_{r, s}, \Delta^{\prime \prime}\right)} q^{\Delta^{\prime \prime}}\right. \\
& \left.\times \sum_{\Delta}[V: L(c, \Delta)] \operatorname{ch} L(c, \Delta)\right]_{q^{0}} \\
& =\left[q^{-1} \sum_{t}\left(q^{b_{t}}-q^{a_{t}}\right) \prod_{n>0}\left(1-q^{n}\right) \sum_{\Delta}[V: L(c, \Delta)] \operatorname{ch} L(c, \Delta)\right]_{q^{0}},
\end{aligned}
$$

where []$_{q^{0}}$ means the coefficient of $q^{0}$. In getting the last expression, we have used diagram (2.9). Now using Corollary 2.5, it is clear that those $\Delta \notin\left\{1-a_{t}, 1-b_{t}\right\}_{t \in \mathbf{Z}}$ will not contribute to $\operatorname{Eul}(V)$. Thus we can write $\operatorname{Eul}(V)$ as

$$
\begin{aligned}
\operatorname{Eul}(V)= & \sum_{t}\left[\sum_{1-b_{s} \rightarrow 1-b_{r}}\left[V: L\left(c, 1-b_{s}\right)\right] q^{-b_{r}}\right. \\
& -\sum_{1-b_{s} \rightarrow 1-a_{r}}\left[V: L\left(c, 1-b_{s}\right)\right] q^{-a_{r}}-\sum_{1-a_{s} \rightarrow 1-b_{r}}\left[V: L\left(c, 1-a_{s}\right)\right] q^{-b_{r}} \\
& \left.+\sum_{1-a_{s} \rightarrow 1-a_{r}}\left[V: L\left(c, 1-a_{s}\right)\right] q^{-b_{r}}\right]_{q^{-b_{t}}}-\sum_{t}[\mathrm{same}]_{q^{-a_{t}}} \\
= & \sum_{1-b_{s} \rightarrow 1-b_{r}}\left[V: L\left(c, 1-b_{s}\right)\right]-\sum_{1-a_{s} \rightarrow 1-b_{r}}\left[V: L\left(c, 1-a_{s}\right)\right] \\
& +\sum_{1-b_{s} \rightarrow 1-a_{r}}\left[V: L\left(c, 1-b_{s}\right)\right]-\sum_{1-a_{s} \rightarrow 1-a_{r}}\left[V: L\left(c, 1-a_{s}\right)\right] \\
= & \sum_{s \in \mathbf{Z}}\left[V: L\left(c, 1-b_{s}\right)\right]\left(\# \text { descendents of } 1-b_{s}\right) \\
& -\sum_{s \in \mathbf{Z}}\left[V: L\left(c, 1-a_{s}\right)\right]\left(\# \text { descendents of } 1-a_{s}\right)
\end{aligned}
$$

We now return to the resolution of $L(c, \Delta)$ given by Proposition 2.4. Using the resolution, we can construct a spectral sequence $\left\{E_{n}\right\}_{n \geq 0}$ according to Proposition A.2. This spectral sequence has

$$
\begin{gathered}
E_{0}^{a, b}=H_{\frac{\infty}{2}+b-a}\left(\operatorname{Vir}, \operatorname{Vir}_{0} ; L\left(c_{p, q}, \Delta_{r, s}\right) \otimes M_{a}\right), \\
E_{1}^{a, b}=H_{a}\left(H_{\frac{\infty}{2}+b-a}\left(\operatorname{Vir}, \operatorname{Vir}_{0} ; L\left(c_{p, q}, \Delta_{r, s}\right) \otimes M_{*}\right), \partial\right),
\end{gathered}
$$


and it converges finitely to a graded object associated with

$$
\begin{gathered}
H_{*}\left(C_{\frac{\infty}{2}+*}\left(\operatorname{Vir}, \operatorname{Vir}_{0} ; L\left(c_{p, q}, \Delta_{r, s}\right) \otimes M_{*}\right), d+\partial\right) \\
\cong H_{\frac{\infty}{2}+*}\left(\operatorname{Vir}, \operatorname{Vir}_{0} ; L\left(c_{p, q}, \Delta_{r, s}\right) \otimes L(c, \Delta)\right) .
\end{gathered}
$$

Of course, it is the right-hand side of (2.31) that we ultimately want to compute. Note that we only need to worry about the case when

$$
c=26-c_{p, q} \text {. }
$$

So from now on, $c$ will assume this value.

Our first step is to compute $E_{0}$. By definition of the $M_{a}$, we have

$$
E_{0}^{a, b} \cong \bigoplus H_{\frac{\infty}{2}+b-a}\left(\operatorname{Vir}, \operatorname{Vir}_{0} ; L\left(c_{p, q}, \Delta_{r, s}\right) \otimes M\left(c, \Delta^{\prime}\right)\right)
$$

$\bigoplus$ here means summing over the Verma modules appearing in $M_{a}$. This reduces the problem to studying the right-hand side of (2.33).

2.1.2. Computing $H_{\frac{\infty}{2}+*}\left(\operatorname{Vir}, \operatorname{Vir}_{0} ; L\left(c_{p, q}, \Delta_{r, s}\right) \otimes M\left(c, \Delta^{\prime}\right)\right)$. By the Reduction Theorem (Theorem 2.13 of [32]), this homology reduces to

$$
H_{\frac{\infty}{2}+*}\left(\operatorname{Vir}, \operatorname{Vir}_{0} ; L\left(c_{p, q}, \Delta_{r, s}\right) \otimes M\left(c, \Delta^{\prime}\right)\right) \cong H_{\frac{\infty}{2}+*}\left(\operatorname{Vir}_{+}, L\left(c_{p, q}, \Delta_{r, s}\right)\right)_{1-\Delta^{\prime}} .
$$

Recall that

$$
\begin{aligned}
& \wedge_{\frac{\infty}{2}+k} \operatorname{Vir}_{-}=\text {Span of the } b_{-n_{1}} b_{-n_{2}} \ldots b_{-n_{k}} 1_{-}, \\
& \wedge_{\frac{\infty}{2}-k} \operatorname{Vir}_{+}=\text {Span of the } c_{-n_{1}} c_{-n_{2}} \ldots c_{-n_{k}} 1_{+} .
\end{aligned}
$$

Thus there is a non-degenerate bilinear pairing

$$
\langle,\rangle: \wedge \frac{\infty}{2}+* \operatorname{Vir}_{-} \times \wedge_{\frac{\infty}{2}-*} \operatorname{Vir}_{+} \rightarrow \mathbf{C}
$$

such that

$$
\left\langle 1_{-}, 1_{+}\right\rangle=1, \quad b_{n}^{\dagger}=b_{-n}, \quad c_{n}^{\dagger}=c_{-n} .
$$

Also we know that $L\left(c_{p, q}, \Delta_{r, s}\right)$ has a non-degenerate bilinear form with

$$
\left\langle v_{\Delta_{r, s}}, v_{\Delta_{r, s}}\right\rangle=1, \quad L_{n}^{\dagger}=L_{-n} .
$$

Note that both forms are non-degenerate on each eigenspace of $L_{0}$. Thus the tensor product of the two forms gives a non-degenerate pairing on the total eigenspaces of $L_{0}$ :

$$
\langle,\rangle: C_{\frac{\infty}{2}+*}\left(\operatorname{Vir}_{-}, L\left(c_{p, q}, \Delta_{r, s}\right)\right)_{\lambda} \times C_{\frac{\infty}{2}-*}\left(\operatorname{Vir}_{+}, L\left(c_{p, q}, \Delta_{r, s}\right)\right)_{\lambda} \rightarrow \mathbf{C}
$$

with

$$
\left\langle 1_{-} \otimes v_{\Delta_{r, s}}, 1_{+} \otimes v_{\Delta_{r, s}}\right\rangle=1, \quad d_{-}^{\dagger}=d_{+},
$$

where $d_{ \pm}$are the respective $\frac{\infty}{2}$-differentials on the two complexes. This pairing induces an isomorphism on homology

$$
H_{\frac{\infty}{2}+*}\left(\operatorname{Vir}_{-}, L\left(c_{p, q}, \Delta_{r, s}\right)\right)_{\lambda} \cong\left[H_{\frac{\infty}{2}-*}\left(\operatorname{Vir}_{+}, L\left(c_{p, q}, \Delta_{r, s}\right)\right)_{\lambda}\right]^{\#} .
$$


Here []$^{\#}$ means the space of linear functionals on []. Thus Eq. (2.34) becomes

$$
\begin{gathered}
H_{\frac{\infty}{2}+*}\left(\operatorname{Vir}, \operatorname{Vir}_{0} ; L\left(c_{p, q}, \Delta_{r, s}\right) \otimes M\left(c, \Delta^{\prime}\right)\right) \\
\cong\left[H_{\frac{\infty}{2}-*}\left(\operatorname{Vir}_{-}, L\left(c_{p, q}, \Delta_{r, s}\right)\right)_{1-\Delta^{\prime}}\right]^{\#} .
\end{gathered}
$$

Since we know the embedding diagram of $M\left(c_{p, q}, \Delta_{r, s}\right)$ (diagram (2.9)), we can use Proposition 2.4 to get a resolution of $L\left(c_{p, q}, \Delta_{r, s}\right)$ :

$$
\begin{aligned}
& \cdots \rightarrow \tilde{M}_{1} \stackrel{\tilde{\partial}}{\rightarrow} \tilde{M}_{0} \stackrel{\tilde{\partial}}{\rightarrow} L\left(c_{p, q}, \Delta_{r, s}\right) \rightarrow 0, \\
& \tilde{M}_{0}=M\left(c_{p, q}, b_{0}\right), \\
& \tilde{M}_{1}=M\left(c_{p, q}, a_{-1}\right) \oplus M\left(c_{p, q}, a_{0}\right), \\
& \tilde{M}_{2}=M\left(c_{p, q}, b_{-1}\right) \oplus M\left(c_{p, q}, b_{1}\right), \quad \text { etc. }
\end{aligned}
$$

We want to use it to compute the homology of $\left\{C_{\frac{\infty}{2}+*}\left(\operatorname{Vir}_{-}, L\left(c_{p, q}, \Delta_{r, s}\right)\right)_{1-\Delta^{\prime}}, d_{-}\right\}$. Once again, Proposition A.2 tells us that we have yet another spectral sequence $\left\{\tilde{E}_{n}\right\}_{n \geq 0}$ with

$$
\begin{aligned}
& \tilde{E}_{0}^{u, v}=H_{\frac{\infty}{2}+v-u}\left(\operatorname{Vir}_{-}, \tilde{M}_{u}\right)_{1-\Delta^{\prime}} \\
& \tilde{E}_{1}^{u, v}=H_{u}\left(H_{\frac{\infty}{2}+v-u}\left(\operatorname{Vir}_{-}, \tilde{M}_{*}\right)_{1-\Delta^{\prime}}, \tilde{\partial}\right),
\end{aligned}
$$

and which converges finitely to a graded object associated with

$$
H_{*}\left(C_{\frac{\infty}{2}+*}\left(\operatorname{Vir}_{-}, \tilde{M}_{*}\right)_{1-\Delta^{\prime}}, d_{-}+\tilde{\partial}\right) \cong H_{\frac{\infty}{2}+*}\left(\operatorname{Vir}_{-}, L\left(c_{p, q}, \Delta_{r, s}\right)\right)_{1-\Delta^{\prime}} .
$$

By definition of $\tilde{M}_{u}$ and Eq. (2.46),

$$
\begin{aligned}
\tilde{E}_{0}^{u, v} \cong \bigoplus_{\substack{d\left(\Delta^{\prime \prime}, \Delta_{r, s}\right)=v \\
\Delta_{r, s \rightarrow \Delta^{\prime \prime}}}} H_{\frac{\infty}{2}+v-u}\left(\operatorname{Vir}_{-}, M\left(c, \Delta^{\prime \prime}\right)\right)_{1-\Delta^{\prime}} \\
\cong \bigoplus_{\substack{d\left(\Delta^{\prime \prime}, \Delta_{r, s}\right)=u \\
\Delta_{r, s} \rightarrow \Delta^{\prime \prime}}} \delta_{v, u} \delta_{1-\Delta^{\prime}, \Delta^{\prime \prime}} \mathbf{C}_{\Delta^{\prime \prime}}
\end{aligned}
$$

We note that the semi-infinite homology of Vir - coincides with the ordinary Lie algebra homology of $\mathrm{Vir}_{-}$. Since the module $M\left(c, \Delta^{\prime \prime}\right)$ is free over $\mathrm{Vir}_{-}$, the homology space is zero except at degree zero. Equation (2.49) immediately implies that the spectral sequence collapses, i.e.

$$
\tilde{E}_{\infty}^{u, v}=\tilde{E}_{0}^{u, v} \cong \delta_{v, u} \bigoplus_{\substack{d\left(\Delta^{\prime \prime}, \Delta_{r, s}\right)=u \\ \Delta_{r, s} \rightarrow \Delta^{\prime \prime}}} \delta_{1, \Delta^{\prime}+\Delta^{\prime \prime}} \mathbf{C}_{\Delta^{\prime \prime}}
$$

Since $\left\{\tilde{E}_{n}\right\}_{n \geq 0}$ converges to a graded object associated with (2.48), we have

$$
H_{\frac{\infty}{2}+v}\left(\operatorname{Vir}_{-}, L\left(c_{p, q}, \Delta_{r, s}\right)\right)_{1-\Delta^{\prime}} \cong \bigoplus_{\substack{d\left(\Delta^{\prime \prime}, \Delta_{r, s}\right)=v \\ \Delta_{r, s} \rightarrow \Delta^{\prime \prime}}} \delta_{1, \Delta^{\prime}+\Delta^{\prime \prime}} \mathbf{C}_{\Delta^{\prime \prime}}
$$

Returning to Eq. (2.34), we have 
Proposition 2.7. For any $\Delta^{\prime}$,

$$
H_{\frac{\infty}{2}+v}\left(\operatorname{Vir}, \operatorname{Vir}_{0} ; L\left(c_{p, q}, \Delta_{r, s}\right) \otimes M\left(26-c_{p, q}, \Delta^{\prime}\right)\right) \cong \bigoplus_{\substack{d\left(\Delta^{\prime \prime}, \Delta_{r, s}\right)=-v \\ \Delta_{r, s} \rightarrow \Delta^{\prime \prime}}} \delta_{1, \Delta^{\prime}+\Delta^{\prime \prime}} \mathbf{C}_{\Delta^{\prime \prime}}
$$

Corollary 2.8 (Theorem 1 [33]).

(a) $H_{\frac{\infty}{2}}\left(\operatorname{Vir}, \operatorname{Vir}_{0} ; L\left(c_{p, q}, \Delta_{r, s}\right) \otimes M\left(26-c_{p, q}, \Delta^{\prime}\right)\right) \neq 0$ iff $\Delta_{r, s} \rightarrow 1-\Delta^{\prime}$.

(b) For $\Delta_{r, s} \rightarrow 1-\Delta^{\prime}$,

$\operatorname{dim} H_{\frac{\infty}{2}+n}\left(\operatorname{Vir}, \operatorname{Vir}_{0} ; L\left(c_{p, q}, \Delta_{r, s}\right) \otimes M\left(26-c_{p, q}, \Delta^{\prime}\right)\right)=\delta_{n+d\left(\Delta_{r, s}, 1-\Delta^{\prime}\right), 0}$.

2.2. Computing $H_{\frac{\infty}{2}+*}\left(\operatorname{Vir}, \operatorname{Vir}_{0} ; L\left(c_{p, q}, \Delta_{r, s}\right) \otimes L(c, \Delta)\right)$

We are now ready to return to Eq. (2.33). Combining Proposition 2.7 and Eq. (2.33), we get

$$
E_{0}^{a, b} \cong \bigoplus_{\substack{d\left(\Delta^{\prime \prime}, \Delta_{r, s}=a-b \\ \Delta_{r, s} \rightarrow \Delta^{\prime \prime}\right.}} \bigoplus^{\prime} \delta_{1, \Delta^{\prime}+\Delta^{\prime \prime}} \mathbf{C}_{\Delta^{\prime \prime}}
$$

Theorem 2.9 (Theorem 2(a) [33]). $H_{\frac{\infty}{2}}\left(\operatorname{Vir}, \operatorname{Vir}_{0} ; L\left(c_{p, q}, \Delta_{r, s}\right) \otimes L\left(26-c_{p, q}, \Delta\right)\right) \neq 0$ iff $\Delta_{r, s} \rightarrow 1-\Delta$.

Proof. Suppose that the $\frac{\infty}{2}$-homology is non-trivial. Recall that $\left\{E_{n}\right\}_{n \geq 0}$ converges finitely to a graded object associated with (2.31). Thus

$$
\begin{aligned}
\operatorname{dim} & H_{\frac{\infty}{2}+b}\left(\operatorname{Vir}, \operatorname{Vir}_{0} ; L\left(c_{p, q}, \Delta_{r, s}\right) \otimes L\left(26-c_{p, q}, \Delta\right)\right) \\
= & \operatorname{dim} H_{b}\left(C_{\frac{\infty}{2}+*}\left(\operatorname{Vir}, \operatorname{Vir}_{0} ; L\left(c_{p, q}, \Delta_{r, s}\right) \otimes M_{*}\right), d+\partial\right) \\
= & \sum_{a} \operatorname{dim} E_{\infty}^{a, b} \\
\leq & \sum_{a} \operatorname{dim} E_{0}^{a, b} \\
= & \sum_{a} \sum_{\substack{d\left(\Delta^{\prime \prime}, \Delta_{r, s}\right)=a-b \\
\Delta_{r, s} \rightarrow \Delta^{\prime \prime}}} \sum \delta_{1, \Delta^{\prime}+\Delta^{\prime \prime}}
\end{aligned}
$$

The third sum ranges over the highest weights $\Delta^{\prime}$ appearing in the $a^{\text {th }}$ term, $M_{a}$, of the resolution of $L\left(26-c_{p, q}, \Delta\right)$ [see Eq. (2.33)]. In particular, these $\Delta^{\prime}$ must be descendents of $\Delta$. By assumption, the left-most term of (2.53) is nonzero. So the rightmost term must be nonzero too. Thus there must be a descendent $\Delta^{\prime}$ of $\Delta\left(\Delta \rightarrow \Delta^{\prime}\right)$ and a descendent $\Delta^{\prime \prime}$ of $\Delta_{r, s}\left(\Delta_{r, s} \rightarrow \Delta^{\prime \prime}\right)$, such that $\Delta^{\prime \prime}=1-\Delta^{\prime}$. This means that $\Delta_{r, s} \rightarrow 1-\Delta^{\prime}$ (at $c_{p, q}$ ). By the reflection principle (2.12), $\Delta \rightarrow \Delta^{\prime}$ (at $26-c_{p, q}$ ) implies that $\left(1-\Delta^{\prime}\right) \rightarrow(1-\Delta)\left(\right.$ at $\left.c_{p, q}\right)$. Combining this with $\Delta_{r, s} \rightarrow\left(1-\Delta^{\prime}\right)$ (at $c_{p, q}$ ), we get $\Delta_{r, s} \rightarrow(1-\Delta)$ (at $\left.c_{p, q}\right)$, proving the first half of our claim. 
Conversely, for $\Delta_{r, s} \rightarrow 1-\Delta$, i.e. $\Delta=1-a_{t}$ or $1-b_{t}$, Lemma 2.6 gives

$$
\begin{aligned}
\operatorname{Eul}(L(c, \Delta))= & \sum_{s}\left[L(c, \Delta): L\left(c, 1-b_{s}\right)\right]\left(\# \text { descendents of } 1-b_{s}\right) \\
& -\sum_{s}\left[L(c, \Delta): L\left(c, 1-a_{s}\right)\right]\left(\# \text { descendents of } 1-a_{s}\right) \\
= & \sum_{s} \delta_{\Delta, 1-b_{s}}\left(\# \text { descendents of } 1-b_{s}\right) \\
& -\sum_{s} \delta_{\Delta, 1-a_{s}}\left(\# \text { descendents of } 1-a_{s}\right) \\
= & \pm(\# \text { descendents of } \Delta)
\end{aligned}
$$

which is non-zero. This completes the proof.

We conclude this section with a more quantitative statement about the above homology. From now on assume that $\Delta_{r, s} \rightarrow 1-\Delta$ at $c_{p, q}$. In this case, Eq. (2.52) can be written as

$$
E_{0}^{a, b} \cong \bigoplus_{\substack{d\left(\Delta^{\prime \prime}, \Delta_{r, s}=a-b \\ \Delta_{r, s} \rightarrow \Delta^{\prime \prime}\right.}} \bigoplus_{\substack{d\left(\Delta, \Delta^{\prime}\right)=a \\ \Delta \rightarrow \Delta^{\prime}}} \delta_{1, \Delta^{\prime}+\Delta^{\prime \prime}} \mathbf{C}_{\Delta^{\prime \prime}}
$$

Recall that $E_{n+1}$ is the homology of the complex:

$$
\cdots \rightarrow E_{n}^{a, b} \rightarrow E_{n}^{a-n-1, b-1} \rightarrow \ldots .
$$

We claim that $E_{n+1}=E_{n}$ for all $n \geq 0$. Consider $n=0$. Using Eq. (2.54) and the reflection principle, we get

$$
\begin{aligned}
\operatorname{dim} E_{0}^{a, b}= & \sum_{\substack{d\left(1-\Delta, \Delta^{\prime}\right)=a, \Delta^{\prime} \rightarrow 1-\Delta \\
d\left(\Delta^{\prime \prime}, \Delta_{r, s}\right)=a-b, \Delta_{r, s} \rightarrow \Delta^{\prime \prime}}} \delta_{\Delta^{\prime}, \Delta^{\prime \prime}} \\
= & \sum_{\substack{d\left(1-\Delta, \Delta^{\prime}\right)=a, d\left(\Delta^{\prime}, \Delta_{r, s}\right)=a-b \\
\Delta_{r, s} \rightarrow \Delta^{\prime} \rightarrow 1-\Delta}} 1 .
\end{aligned}
$$

Note that if $\Delta_{r, s} \rightarrow \Delta^{\prime} \rightarrow 1-\Delta$, then

$$
d\left(1-\Delta, \Delta_{r, s}\right)=d\left(1-\Delta, \Delta^{\prime}\right)+d\left(\Delta^{\prime}, \Delta_{r, s}\right) .
$$

Thus (2.56) is zero unless

$$
d\left(1-\Delta, \Delta_{r, s}\right)=2 a-b
$$

In particular, we have

$$
\operatorname{dim} E_{0}^{a, b} \neq 0 \Rightarrow \operatorname{dim} E_{0}^{a-1, b-1}=0 .
$$

This means that (see sequence (2.55))

$$
E_{1}=E_{0} .
$$

By the same argument, we see that

$$
E_{n}=E_{0}, \quad n>0 .
$$


So Eq. (2.53) becomes an equality:

$$
\operatorname{dim} H_{\frac{\infty}{2}+b}\left(\operatorname{Vir}, \operatorname{Vir}_{0} ; L\left(c_{p, q}, \Delta_{r, s}\right) \otimes L\left(26-c_{p, q}, \Delta\right)\right)
$$

Proposition 2.10 (Theorem 2(b) [33]). For $\Delta_{r, s} \rightarrow 1-\Delta$,

$$
\begin{aligned}
\operatorname{dim} & H_{\frac{\infty}{2}+b}\left(\operatorname{Vir}, \operatorname{Vir}_{0} ; L\left(c_{p, q}, \Delta_{r, s}\right) \otimes L\left(26-c_{p, q}, \Delta\right)\right) \\
\quad= & \begin{cases}1+(-1)^{b+d\left(\Delta_{r, s}, 1-\Delta\right)}-\delta_{|b|, d\left(\Delta_{r, s}, 1-\Delta\right)} & \text { if }|b| \leq d\left(\Delta_{r, s}, 1-\Delta\right) \\
0 & \text { otherwise }\end{cases}
\end{aligned}
$$

Proof. Let's abbreviate the left-hand side of Eq. (2.63) as $B_{b}$ and write $k=d\left(\Delta_{r, s}\right.$, $1-\Delta$ ). From the conditions of the summation in Eq. (2.62), it's clear that for $B_{b} \neq 0$, we must have

$$
\begin{gathered}
k \geq d\left(1-\Delta, \Delta^{\prime}\right)=a \geq 0 \\
k \geq d\left(\Delta^{\prime}, \Delta_{r, s}\right)=a-b \geq 0 .
\end{gathered}
$$

They imply that

$$
|b| \leq k
$$

which proves half of Eq. (2.63). Assume the inequality (2.66). Equation (2.62) says that $B_{b} \neq 0$ implies that $2 a=k+b$ is even. Let's assume so. Then Eq. (2.62) becomes

$$
B_{b}=\sum_{\substack{d\left(1-\Delta, \Delta^{\prime}\right)=\frac{k+b}{2}, d\left(\Delta^{\prime}, \Delta_{r, s}\right)=\frac{k-b}{2} \\
\Delta_{r, s} \rightarrow \Delta^{\prime} \rightarrow 1-\Delta}} 1=\left\{\begin{array}{lll}
1 & \text { if } & |b|=k \\
2 & \text { if } & |b|<k .<.
\end{array}\right.
$$

\section{Original Problems}

\subsection{Returning to Problem 1}

We are now ready to determine exactly which $F_{\xi, \eta}$ affords non-trivial homology. Once again, the balance of central charge fixes $\sqrt{-1} \xi$ to be

$$
\sqrt{-1} \xi=\frac{p+q}{\sqrt{2 p q}}
$$

Theorem 3.1 (Theorem 3(a) [33]). For $\eta \in \mathbf{C}, H_{\frac{\infty}{2}}\left(\operatorname{Vir}, \operatorname{Vir}_{0} ; L\left(c_{p, q}, \Delta_{r, s}\right) \otimes F_{\xi, \eta}\right) \neq 0$ iff

$$
\eta=\sqrt{-1} \varepsilon_{1} \frac{\left(2 p q t+p r+\varepsilon_{2} q s\right)}{\sqrt{2 p q}} \text { for some } t \in \mathbf{Z}, \varepsilon_{1}, \varepsilon_{2}= \pm 1 \text {. }
$$

Proof. Computing the formal character of $F_{\xi, \eta}$, we get

$$
\begin{aligned}
\operatorname{ch} F_{\xi, \eta} & \stackrel{\text { def }}{=} \operatorname{tr}_{F_{\xi, \eta}} q^{L_{0}-c / 24} \\
& =q^{-\left(1-12 \xi^{2}\right) / 24} q^{\frac{1}{2}\left(\eta^{2}-\xi^{2}\right)} \prod_{n>0}\left(1-q^{n}\right)^{-1} \\
& =\operatorname{ch} M\left(1-12 \xi^{2}, \frac{1}{2}\left(\eta^{2}-\xi^{2}\right)\right) .
\end{aligned}
$$


Suppose

$$
\eta= \pm \sqrt{-1} \frac{(2 p q t+p r+\varepsilon q s)}{\sqrt{2 p q}}
$$

This gives

$$
\frac{1}{2}\left(\eta^{2}-\xi^{2}\right)=\left\{\begin{array}{lll}
1-a_{t} & \text { if } & \varepsilon=+1 \\
1-b_{t} & \text { if } & \varepsilon=-1
\end{array}\right.
$$

Using this to compute the Euler characteristic, we get

$$
\begin{aligned}
\text { Eul } F_{\xi, \eta} & =\left\{\begin{array}{lll}
(-1)^{d\left(a_{t}, \Delta_{r, s}\right)} & \text { if } & \varepsilon=+1 \\
(-1)^{d\left(b_{t}, \Delta_{r, s}\right)} & \text { if } & \varepsilon=-1
\end{array}\right. \\
& =\varepsilon
\end{aligned}
$$

In particular

$$
H_{\frac{\infty}{2}}\left(\operatorname{Vir}, \operatorname{Vir}_{0} ; L\left(c_{p, q}, \Delta_{r, s}\right) \otimes F_{\xi, \eta}\right) \neq 0 .
$$

Conversely suppose (3.6), i.e. $F_{\xi, \eta}$ affords homology. This implies that $F_{\xi, \eta}$ has a composition factor that affords homology, i.e.

$$
H_{\frac{\infty}{2}}\left(\operatorname{Vir}, \operatorname{Vir}_{0} ; L\left(c_{p, q}, \Delta_{r, s}\right) \otimes L\left(26-c_{p, q}, \Delta\right)\right) \neq 0
$$

for some $\Delta$ with

$$
\left[F_{\xi, \eta}: L\left(26-c_{p, q}, \Delta\right)\right] \neq 0 .
$$

By Theorem 2.9, (3.7) implies that

$$
\Delta_{r, s} \rightarrow(1-\Delta) \quad \text { at } \quad c_{p, q} .
$$

By Eqs. (3.2) and (3.8), we have

$$
\left[M\left(26-c_{p, q}, \frac{1}{2}\left(\eta^{2}-\xi^{2}\right)\right): L\left(26-c_{p, q}, \Delta\right)\right] \neq 0 .
$$

This means that (Lemma 2.3)

$$
\frac{1}{2}\left(\eta^{2}-\xi^{2}\right) \rightarrow \Delta \quad \text { at } \quad 26-c_{p, q}
$$

or, by the reflection principle (2.12),

$$
(1-\Delta) \rightarrow\left(1-\frac{1}{2}\left(\eta^{2}-\xi^{2}\right)\right) \quad \text { at } \quad c_{p, q} .
$$

Combining (3.9) and (3.12), we have

$$
\Delta_{r, s} \rightarrow\left(1-\frac{1}{2}\left(\eta^{2}-\xi^{2}\right)\right) \quad \text { at } \quad c_{p, q} .
$$

This means that (diagram (2.9))

$$
1-\frac{1}{2}\left(\eta^{2}-\xi^{2}\right)=a_{t} \quad \text { or } \quad b_{t} \quad \text { for some } t .
$$

Solving this gives us Eq. (3.3).

Theorem 3.1 answers Problem 1. 


\subsection{Multiplicities of Physical States (Problem 2)}

In the last section, we had quite a bit of success in computing $\frac{\infty}{2}$-homology, when the coefficients involved only irreducibles or Verma modules. Those computations relied heavily on constructing resolutions. In turn, constructing a resolution requires the knowledge of the coefficient module structure. Now in Problem 2, we are dealing with

$$
H_{\frac{\infty}{2}+*}\left(\operatorname{Vir}, \operatorname{Vir}_{0} ; L\left(c_{p, q}, \Delta_{r, s}\right) \otimes F_{\xi, \eta}\right)=?
$$

where

$$
\sqrt{-1} \xi=\frac{p+q}{\sqrt{2 p q}}, \quad \sqrt{-1} \eta=\sigma \frac{(2 p q t+p r+\varepsilon q s)}{\sqrt{2 p q}}, \quad \sigma, \varepsilon= \pm 1, t \in \mathbf{Z} .
$$

We can try to resolve $L\left(c_{p, q}, \Delta_{r, s}\right)$ by Verma modules as before. But then we would end up with something like $H_{\frac{\infty}{2}+*}\left(\mathrm{Vir}_{+}, F_{\xi, \eta}\right)$. Computing this directly requires knowing the structure of $F_{\xi, \eta}$ to a great extent. There is, fortunately, a slicker way to do (3.15). There exists yet another object that resolves $L\left(c_{p, q}, \Delta_{r, s}\right)$. It is known as the Felder resolution [20]. It is a chain complex $\left\{F_{\xi^{\prime}, \eta_{*}}, \delta\right\}$ consisting of Feigin-Fuchs modules

$$
\cdots \rightarrow F_{\xi^{\prime}, \eta_{1}} \stackrel{\delta}{\rightarrow} F_{\xi^{\prime}, \eta_{0}} \stackrel{\delta}{\rightarrow} F_{\xi^{\prime}, \eta_{-1}} \rightarrow \cdots
$$

with

$$
\begin{aligned}
\xi^{\prime} & =\frac{p-q}{\sqrt{2 p q}}, \\
\eta_{-2 t^{\prime}} & =\frac{2 p q t^{\prime}+p r-p s}{\sqrt{2 p q}}, \\
\eta_{-2 t^{\prime}-1} & =\frac{2 p q t^{\prime}+p r+q s}{\sqrt{2 p q}}, \quad t^{\prime} \in \mathbf{Z},
\end{aligned}
$$

and having homology that is isomorphic to $L\left(c_{p, q}, \Delta_{r, s}\right)$ :

$$
H_{n}\left(F_{\xi^{\prime}, \eta_{*}}, \delta\right) \cong \delta_{n, 0} L\left(c_{p, q}, \Delta_{r, s}\right) .
$$

We now apply this resolution to study the homology (3.15). By Proposition A.2, there is a spectral sequence $\left\{E_{n}\right\}_{n \geq 0}$ with

$$
\begin{aligned}
& E_{0}^{a, b}=H_{\frac{\infty}{2}+b-a}\left(\operatorname{Vir}, \operatorname{Vir}_{0} ; F_{\xi^{\prime}, \eta_{a}} \otimes F_{\xi, \eta}\right), \\
& E_{1}^{a, b}=H_{a}\left(H_{\frac{\infty}{2}+b-a}\left(\operatorname{Vir}, \operatorname{Vir}_{0} ; F_{\xi^{\prime}, \eta_{*}} \otimes F_{\xi, \eta}\right), \partial\right),
\end{aligned}
$$

and which converges finitely to the graded object associated with

$$
\begin{aligned}
& H_{*}\left(C_{\frac{\infty}{2}+*}\left(\operatorname{Vir}, \operatorname{Vir}_{0} ; F_{\xi^{\prime}, \eta_{*}} \otimes F_{\xi, \eta}\right), d+\partial\right) \\
& \quad \cong H_{\frac{\infty}{2}+*}\left(\operatorname{Vir}, \operatorname{Vir}_{0} ; L\left(c_{p, q}, \Delta_{r, s}\right) \otimes F_{\xi, \eta}\right) .
\end{aligned}
$$

( $\partial$ is the map induced by Felder's map $\delta$.) Of course the right-hand side of (3.22) is what we eventually want to know. But we must first study the spectral sequence.

3.2.1. Computing $E_{0}$. At first sight, we seem to have made matters worse by introducing the resolution (3.17), because we have ended up with (3.20), which involves the uncanny object $F_{\xi^{\prime}, \eta_{a}} \otimes F_{\xi, \eta}$ ! However, this tensor product module over Vir turns 
out to have a remarkable property: it is invariant under the group $S O(2, \mathrm{C})$. In fact, for any $\xi, \eta, \xi^{\prime}, \eta^{\prime} \in \mathbf{C}$ and

$$
\left(\begin{array}{cc}
f & i g \\
-i g & f
\end{array}\right) \in S O(2, \mathbf{C}), \quad\left(f^{2}-g^{2}=1\right)
$$

we have a canonical isomorphism of Vir-modules

$$
F_{\xi^{\prime}, \eta^{\prime}} \otimes F_{\xi, \eta}=F_{\mu^{\prime}, \nu^{\prime}} \otimes F_{\mu, \nu}
$$

where

$$
\begin{aligned}
& \left(\begin{array}{l}
\mu^{\prime} \\
\mu
\end{array}\right)=\left(\begin{array}{cc}
f & i g \\
-i g & f
\end{array}\right)\left(\begin{array}{l}
\xi^{\prime} \\
\xi
\end{array}\right), \\
& \left(\begin{array}{c}
\nu^{\prime} \\
\nu
\end{array}\right)=\left(\begin{array}{cc}
f & i g \\
-i g & f
\end{array}\right)\left(\begin{array}{l}
\eta^{\prime} \\
\eta
\end{array}\right) .
\end{aligned}
$$

This symmetry was observed by one of us [43] in studying the problem of computing $H_{\frac{\infty}{2}+*}\left(\operatorname{Vir}, \operatorname{Vir}_{0} ; V(\mathbf{p}, \boldsymbol{\mu})\right)$, where $V(\mathbf{p}, \boldsymbol{\mu})$ is the usual bosonic Fock space with fixed momentum $\mathbf{p}$ in the $D$-dimensional string theory. The Virasoro algebra operates in $V(\mathbf{p}, \boldsymbol{\mu})$ by a deformed stress-energy:

$$
T(z)=\frac{1}{2}: \mathbf{j}(z) \cdot \mathbf{j}(z):+\boldsymbol{\mu} \cdot \partial \mathbf{j}(z),
$$

where $\mathbf{j}(z)$ is the $D$-dimensional bosonic field with $\mathbf{j}_{0}=\mathbf{p}$. Observe that for $D=2$, $V(\mathbf{p}, \boldsymbol{\mu})$ is canonically isomorphic to (3.23) with $\mathbf{v}=\boldsymbol{\mu}-\mathbf{p}$.

Equation (3.23) will be our key to computing $E_{0}$ [Eq. (3.20)]. It is well-known that at generic values of $\mu, \nu$, the Feigin-Fuchs module $F_{\mu, \nu}$ is isomorphic to the Verma module $M\left(1-12 \mu^{2}, \frac{1}{2}\left(\nu^{2}-\mu^{2}\right)\right)$. In this case, both modules are irreducible. But what we have is $F_{\xi^{\prime}, \eta_{a}} \otimes F_{\xi, \eta}$, where the parameters are given by Eqs. (3.16), (3.18). Neither factor of the tensor product is generic. Thus we hope that we can use an element of $S O(2, \mathbf{C})$ to rotate this tensor product [according to Eqs. (3.23), (3.24)] to $F_{\mu^{\prime}, \nu^{\prime}} \otimes F_{\mu, \nu}$, such that both new factors become generic. If such a rotation exists, then computing $E_{0}$ becomes possible:

$$
\begin{aligned}
E_{0}^{a, b} & =H_{\frac{\infty}{2}+b-a}\left(\operatorname{Vir}, \operatorname{Vir}_{0} ; F_{\xi^{\prime}, \eta_{a}} \otimes F_{\xi, \eta}\right) \\
& \cong H_{\frac{\infty}{2}+b-a}\left(\operatorname{Vir}_{\operatorname{Vir}_{0}} ; F_{\mu^{\prime}, \nu^{\prime}} \otimes F_{\mu, \nu}\right) \\
& \cong H_{\frac{\infty}{2}+b-a}\left(\operatorname{Vir}, \operatorname{Vir}_{0} ; M\left(1-12 \mu^{\prime 2}, \frac{1}{2}\left(\nu^{\prime 2}-\mu^{\prime 2}\right)\right) \otimes M\left(1-12 \mu^{2}, \frac{1}{2}\left(\nu^{2}-\mu^{2}\right)\right)\right) \\
& \cong H_{\frac{\infty}{2}+b-a}\left(\operatorname{Vir}_{+}, M\left(1-12 \mu^{\prime 2}, \frac{1}{2}\left(\nu^{\prime 2}-\mu^{\prime 2}\right)\right)\right)_{1-\frac{1}{2}\left(\nu^{2}-\mu^{2}\right)} \quad \text { (Reduction Thm) } \\
& \cong\left[H_{\frac{\infty}{2}+a-b}\left(\operatorname{Vir}_{-}, M\left(1-12 \mu^{\prime 2}, \frac{1}{2}\left(\nu^{\prime 2}-\mu^{\prime 2}\right)\right)\right)\right]_{1-\frac{1}{2}\left(\nu^{2}-\mu^{2}\right)}^{\#} \quad \text { (taking dual) } \\
& \cong \delta_{a, b} \mathbf{C} \delta_{\frac{1}{2}\left(\nu^{2}-\mu^{\prime 2}\right), 1-\frac{1}{2}\left(\nu^{2}-\mu^{2}\right)} .
\end{aligned}
$$

Therefore, we must determine whether or not the above rotation exists.

Proposition 3.2. Let

$$
\begin{gathered}
\xi^{\prime}=\frac{p-q}{\sqrt{2 p q}}, \quad \eta^{\prime}=\frac{2 p q t^{\prime}+p r+\varepsilon^{\prime} q s}{\sqrt{2 p q}} \\
\sqrt{-1} \xi=\frac{p+q}{\sqrt{2 p q}}, \quad \sqrt{-1} \eta=-\frac{2 p q t+p r+\varepsilon q s}{\sqrt{2 p q}}
\end{gathered}
$$


Then there exists

$$
\left(\begin{array}{cc}
f & i g \\
-i g & f
\end{array}\right) \in S O(2, \mathbf{C})
$$

such that on the right-hand side of Eq. (3.23), both $F_{\mu^{\prime}, \nu^{\prime}}, F_{\mu, \nu}$ are irreducible.

Proof. Note that the statement would be false if we had chosen a different sign for $\sqrt{-1} \eta$ (see below). Choose $f$ to be a transcendental number. We will show that $F_{\mu, \nu}$ is irreducible. The argument is the same for $F_{\mu^{\prime} . \nu^{\prime}}$.

Recall that $F_{\mu, \nu}$ is irreducible iff the corresponding Verma module $M\left(1-12 \mu^{2}\right.$, $\left.\frac{1}{2}\left(\nu^{2}-\mu^{2}\right)\right)$ is irreducible. But the latter is determined by whether the Kac determinant has a zero. This determinant is the product of expressions of the form

$$
\phi_{\alpha, \beta}(c, \Delta)=B_{\alpha, \beta}(\mu, \nu) \cdot B_{-\alpha,-\beta}(\mu, \nu), \quad \alpha, \beta \in \mathbf{N},
$$

where

$$
\begin{gathered}
c=1-12 \mu^{2}, \quad \Delta=\frac{1}{2}\left(\nu^{2}-\mu^{2}\right), \\
B_{\alpha, \beta}(\mu, \nu)=(\alpha \mu-\nu)(\beta \mu-\nu)-\frac{(\alpha-\beta)^{2}}{2} .
\end{gathered}
$$

To show that $M(c, \Delta)$ is irreducible, it is enough to have

$$
B_{\alpha, \beta}(\mu, \nu) \neq 0 \quad \text { for all integers } \alpha, \beta .
$$

By Eq. (3.24) and a simple calculation, we get

$$
\begin{aligned}
B_{\alpha, \beta}(\mu, \nu)= & B_{\alpha, \beta}\left(-i g \xi^{\prime}+f \xi,-i g \eta^{\prime}+f \eta\right) \\
= & f^{2}\left(B_{\alpha, \beta}(\xi, \eta)-B_{\alpha, \beta}\left(\xi^{\prime}, \eta^{\prime}\right)\right) \\
& -f g \Lambda+B_{\alpha, \beta}\left(\xi^{\prime}, \eta^{\prime}\right)
\end{aligned}
$$

where

$$
\Lambda=2 \alpha \beta \xi^{\prime} i \xi+2 \eta^{\prime} i \eta-(\alpha+\beta)\left(\xi^{\prime} i \eta+\eta^{\prime} i \xi\right) .
$$

For the given values of parameters $\xi^{\prime}, \eta^{\prime}, \xi, \eta$, it is obvious that $B_{\alpha, \beta}(\xi, \eta)$, $B_{\alpha, \beta}\left(\xi^{\prime}, \eta^{\prime}\right)$ and $\Lambda$ are rational whenever $\alpha, \beta \in \mathbf{Z}$. Suppose

$$
B_{\alpha, \beta}(\mu, \nu)=0 \quad \text { for some } \alpha, \beta \in \mathbf{Z} .
$$

We wish to get a contradiction. Since $f$ is trancendental (hence, so is $g$ ), Eq. (3.32) implies that

$$
\begin{aligned}
B_{\alpha, \beta}(\xi, \eta) & =0 \\
B_{\alpha, \beta}\left(\xi^{\prime}, \eta^{\prime}\right) & =0 \\
\Lambda & =0 .
\end{aligned}
$$

Substituting the (3.28) into (3.35), we get

$$
\begin{aligned}
p \alpha+q \beta-\sqrt{2 p q} i \eta & =0, \\
\text { or } \quad p \beta+q \alpha-\sqrt{2 p q} i \eta & =0 .
\end{aligned}
$$

Substituting (3.27) into (3.36), we get

$$
\begin{aligned}
p \alpha-q \beta-\sqrt{2 p q} \eta^{\prime} & =0, \\
\text { or } \quad p \beta-q \alpha-\sqrt{2 p q} \eta^{\prime} & =0 .
\end{aligned}
$$


Case 1. Equations (3.37), (3.38), (3.40) hold. Solving for $\beta$, we get

$$
\begin{aligned}
2 q \beta & =-\left(\eta^{\prime}-i \eta\right) \sqrt{2 p q} \\
& =-\left(2 p q t^{\prime}+p r+\varepsilon^{\prime} q s+2 p q t+p r+\varepsilon q s\right) .
\end{aligned}
$$

The right-hand side is not divisible by $2 q$ (because $\operatorname{gcd}(p, q)=1,0<r<q$ ), while the left-hand side is, which is a contradiction [Eq. (3.42) would have been all right if i $\eta$ had a different sign!].

Case 2. Equations (3.37), (3.38), (3.41) hold. Then substituting the given $\xi, \xi^{\prime}$ and

$$
\begin{aligned}
& \sqrt{2 p q} i \eta=p \alpha+q \beta \\
& \sqrt{2 p q} \eta^{\prime}=p \beta-q \alpha
\end{aligned}
$$

into (3.37) and simplifying it, we get

$$
\left(p^{2}-q^{2}\right)(\alpha-\beta)^{2}=0
$$

which implies

$$
\alpha=\beta \text {. }
$$

Combining (3.43), (3.44), (3.46), we get

$$
(p-q) i \eta=(p+q) \eta^{\prime} \text {. }
$$

Using (3.28), we get

$$
p\left(p q\left(t+t^{\prime}\right)+p r+\frac{\varepsilon+\varepsilon^{\prime}}{2} q s\right)=q\left(p q\left(t-t^{\prime}\right)+\frac{\varepsilon-\varepsilon^{\prime}}{2} q s\right) .
$$

Once again, the right-hand side is divisible by $q$ while the left-hand side isn't.

Case 3. Equations (3.37), (3.39), (3.40) hold. Just like Case 2.

Case 4. Equations (3.37), (3.39), (3.41) hold. Just like Case 1.

In any case, Eq. (3.34) is impossible. This completes the proof.

We now return to $E_{0}$ [Eq. (3.26)] for $\xi, \eta, \xi^{\prime}, \eta^{\prime}$ given by Eqs. (3.27), (3.28). Proposition 3.2 tells us that Eq. (3.26) holds. By (3.24), it is easy to check that

$$
\begin{aligned}
& \nu^{\prime 2}+\nu^{2}=\eta^{2}+\eta^{2}, \\
& \mu^{2}+\mu^{2}=\xi^{\prime 2}+\xi^{2} .
\end{aligned}
$$

Thus Eq. (3.26) becomes (with $\eta^{\prime}=\eta_{a}$ )

$$
E_{0}^{a, b} \cong \delta_{a, b} \delta_{1, \frac{1}{2}\left(\eta_{a}^{2}+\eta^{2}-\xi^{2}-\xi^{2}\right)} \mathbf{C} .
$$

Recall that we defined the distance function $d\left(\Delta, \Delta^{\prime}\right)$ for $\Delta, \Delta^{\prime}$ belonging to the same Verma module embedding diagram. Let's try to rewrite Eq. (3.51) in terms of this function. It is easy to check that

$$
\begin{aligned}
1-12 \xi^{\prime 2} & =c_{p, q}, \\
\frac{1}{2}\left(\eta_{k}^{2}-\xi^{\prime 2}\right) & =\left\{\begin{array}{llll}
b_{-\frac{k}{2}} & \text { if } & k & \text { even } \quad\left(\varepsilon^{\prime}=-1\right) \\
a_{-\frac{k+1}{2}} & \text { if } & k & \text { odd } \quad\left(\varepsilon^{\prime}=1\right)
\end{array}\right. \\
1-12 \xi^{2} & =26-c_{p, q}, \\
\frac{1}{2}\left(\eta^{2}-\xi^{2}\right) & =\left\{\begin{array}{lll}
1-b_{t} & \text { if } & \varepsilon=-1 \\
1-a_{t} & \text { if } & \varepsilon=1
\end{array}\right.
\end{aligned}
$$


Thus

$$
\begin{aligned}
& 1=\frac{1}{2}\left(\eta^{2}+\eta_{k}^{2}-\xi^{2}-\xi^{2}\right) \text { iff } \\
& k=\left\{\begin{array}{ll}
-2 t & \text { if } \varepsilon=-1 \\
-2 t-1 & \text { if } \varepsilon=1
\end{array}\right. \text { iff } \\
& k=\left\{\begin{array}{l}
-\operatorname{sign}(t) d\left(b_{t}, b_{0}\right) \quad \text { if } \varepsilon=-1 \\
-\operatorname{sign}(t) d\left(a_{t}, b_{0}\right) \text { if } \varepsilon=1
\end{array} \quad\right. \text { iff } \\
& k=-\operatorname{sign}(t) d\left(\frac{1}{2}\left(\eta^{2}-\xi^{2}\right), 1-b_{0}\right) .
\end{aligned}
$$

The last part follows from the reflection image of diagram (2.9) and Eq. (3.52). Let's write

$$
\begin{aligned}
\pi(\eta) & =\operatorname{sign}(-\sqrt{-1} \eta)=\operatorname{sign}(t) \\
d_{\eta} & =d\left(\frac{1}{2}\left(\eta^{2}-\xi^{2}\right), 1-b_{0}\right)
\end{aligned}
$$

Then

$$
\delta_{1, \frac{1}{2}\left(\eta^{2}+\eta_{k}^{2}-\xi^{2}-\xi^{2}\right)}=\delta_{k+\pi(\eta) d_{\eta}, 0} .
$$

Now Eq. (3.51) becomes

$$
E_{0}^{a, b} \cong \delta_{a, b} \delta_{a+\pi(\eta) d_{\eta}, 0} \mathbf{C}
$$

This completes the computation for $E_{0}^{a, b}(3.20)$.

3.2.2. Returning to $H_{\frac{\infty}{2}+*}\left(\operatorname{Vir}, \operatorname{Vir}_{0} ; L\left(c_{p, q}, \Delta_{r, s}\right) \otimes F_{\xi, \eta}\right)$. Equation (3.57) immediately implies that the spectral sequence $\left\{E_{n}\right\}_{n \geq 0}$ collapses, i.e.

$$
E_{\infty}^{a, b}=E_{0}^{a, b} \cong \delta_{a, b} \delta_{b+\pi(\eta) d_{\eta}, 0} \mathbf{C} .
$$

Now, the spectral sequence also converges finitely to the graded object associated with

$$
H_{*}\left(C_{\frac{\infty}{2}+*}\left(\operatorname{Vir}, \operatorname{Vir}_{0} ; F_{\xi^{\prime}, \eta_{*}} \otimes F_{\xi, \eta}\right), d+\partial\right) \text {. }
$$

Therefore

$$
H_{n}\left(C_{\frac{\infty}{2}+*}\left(\operatorname{Vir}, \operatorname{Vir}_{0} ; F_{\xi^{\prime}, \eta_{*}} \otimes F_{\xi, \eta}\right), d+\partial\right) \cong \delta_{n+\pi(\eta) d_{\eta}, 0} \mathbf{C} .
$$

Using Eq. (3.22), we get

$$
H_{\frac{\infty}{2}+n}\left(\operatorname{Vir}, \operatorname{Vir}_{0} ; L\left(c_{p, q}, \Delta_{r, s}\right) \otimes F_{\xi, \eta}\right) \cong \delta_{n+\pi(\eta) d_{\eta}, 0} \mathbf{C}
$$
Theorem 3.3 (Theorem 3(b) [33]). For $\sqrt{-1} \xi=\frac{p+q}{\sqrt{2 p q}}, \sqrt{-1} \eta=\delta \frac{2 p q t+p r+\varepsilon q s}{\sqrt{2 p q}}$
$t \in \mathbf{Z}, \sigma, \varepsilon= \pm 1$,

$$
H_{\frac{\infty}{2}+n}\left(\operatorname{Vir}, \operatorname{Vir}_{0} ; L\left(c_{p, q}, \Delta_{r, s}\right) \otimes F_{\xi, \eta}\right) \cong \delta_{n+\pi(\eta) d_{\eta}, 0} \mathbf{C} .
$$

Proof. We established Eq. (3.61) using Proposition 3.2, which holds only for $\sigma=-1$. For $\sigma=+1$, observe that there is a canonical isomorphism of Vir-modules,

$$
F_{\xi, \eta} \cong F_{\xi,-\eta}^{\#} \quad\left(\text { restricted dual of } F_{\xi,-\eta}\right) \text {. }
$$

This means that there is a non-degenerate bilinear pairing

$$
\begin{aligned}
\langle,\rangle & : C_{\frac{\infty}{2}+n}\left(\operatorname{Vir}, \operatorname{Vir}_{0} ; L\left(c_{p, q}, \Delta_{r, s}\right) \otimes F_{\xi, \eta}\right) \\
& \times C_{\frac{\infty}{2}-n}\left(\operatorname{Vir}, \operatorname{Vir}_{0} ; L\left(c_{p, q}, \Delta_{r, s}\right) \otimes F_{\xi,-\eta}\right) \rightarrow \mathbf{C}
\end{aligned}
$$


such that $d^{\dagger}=d$. This induces a similar pairing on homology. Thus

$$
\begin{aligned}
& H_{\frac{\infty}{2}+n}\left(\operatorname{Vir}, \operatorname{Vir}_{0} ; L\left(c_{p, q}, \Delta_{r, s}\right) \otimes F_{\xi, \eta}\right) \\
& \quad \cong H_{\frac{\infty}{2}-n}\left(\operatorname{Vir}, \operatorname{Vir}_{0} ; L\left(c_{p, q}, \Delta_{r, s}\right) \otimes F_{\xi,-\eta}\right)^{\#} .
\end{aligned}
$$

But now $F_{\xi,-\eta}$ corresponds to $\sigma=-1$, for which Eq. (3.61) holds. So Eq. (3.65) becomes

$$
H_{\frac{\infty}{2}+n}\left(\operatorname{Vir}, \operatorname{Vir}_{0} ; L\left(c_{p, q}, \Delta_{r, s}\right) \otimes F_{\xi, \eta}\right) \cong \delta_{-n+\pi(-\eta) d_{-\eta}, 0} \mathbf{C} \cong \delta_{-n-\pi(\eta) d_{\eta}, 0} \mathbf{C} .
$$

Theorem 3.3 answers Problem 2. The diagrams below show a few examples of BRST invariant states with $\sqrt{-1} \eta>0$. Each dot represents a single BRST invariant state whose $(x, y)$-coordinates are the "quantum numbers," $(\sqrt{2 p q} i \eta,-g h \#)$, corresponding to that state.

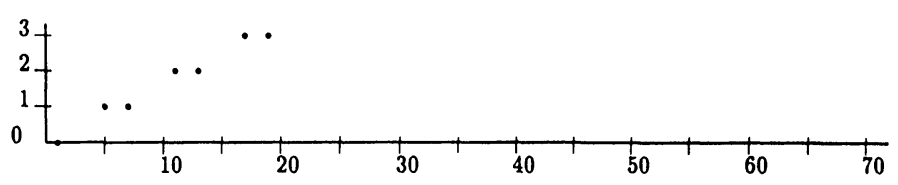

Fig. 1. $(p, q)=(3,2)$ Pure gravity

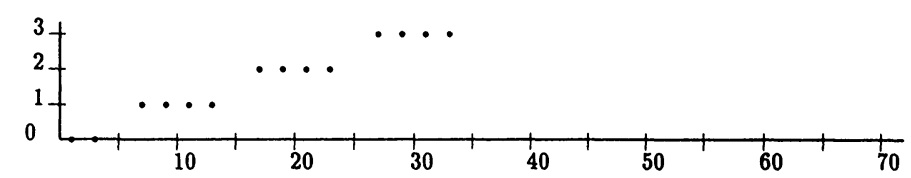

Fig. 2. $(p, q)=(5,2)$ Yang-Lee edge singularity

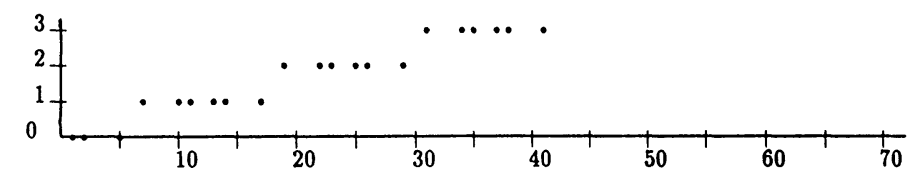

Fig. 3. $(p, q)=(4,3)$ Ising

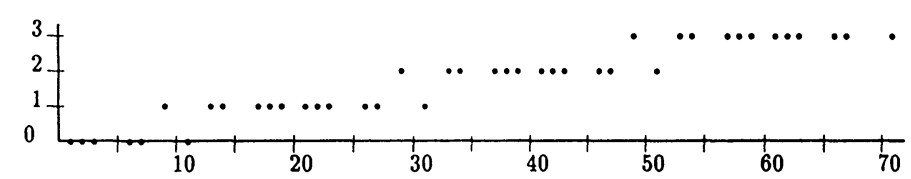

Fig. 4. $(p, q)=(5,4)$ Tri-critical Ising

\subsection{Formula for the BRST Invariant States}

In the last section, we computed the $\frac{\infty}{2}$-homology by getting a double complex from Felder's resolution. We then applied a rotation symmetry to show that the resulting 
spectral sequence collapses at $E_{0}$. This led to the isomorphisms [Eqs. (3.22), (3.60) with $\pi(\eta)=-1$ ]

$$
\begin{aligned}
\delta_{n, d_{\eta}} \mathbf{C} & \cong H_{n}\left(C_{\frac{\infty}{2}+*}\left(\operatorname{Vir}, \operatorname{Vir}_{0} ; F_{\xi^{\prime}, \eta_{*}} \otimes F_{\xi, \eta}\right), d+\partial\right) \\
& \cong H_{\frac{\infty}{2}+n}\left(\operatorname{Vir}, \operatorname{Vir}_{0} ; L\left(c_{p, q}, \Delta_{r, s}\right) \otimes F_{\xi, \eta}\right) .
\end{aligned}
$$

Because of the degenerate nature of the homology of the double complex $\left\{C_{\frac{\infty}{2}+*}(\mathrm{Vir}\right.$, $\left.\left.\operatorname{Vir}_{0} ; F_{\xi^{\prime}, \eta_{*}} \otimes F_{\xi, \eta}\right), d+\partial\right\}$, there is a trick in homology algebra which allows one to get a formula for a non-trivial $d$-cycle (i.e. non-trivial BRST invariant state). It is known as the "zig-zag method" [2], and it goes as follows.

We will illustrate the case in which $i \eta=\sigma \frac{2 p q t+p r+\varepsilon q s}{\sqrt{2 p q}}, \sigma=-1$. The case $\sigma=1$ has a similar argument. Recall that [Eqs. (3.20), (3.51), 3.57)]

$$
H_{\frac{\infty}{2}+k}\left(\operatorname{Vir}, \operatorname{Vir}_{0} ; F_{\xi^{\prime}, \eta_{l}} \otimes F_{\xi, \eta}\right) \cong \mathbf{C} \delta_{k, 0} \delta_{1, \frac{1}{2}\left(\eta_{l}^{2}+\eta^{2}-\xi^{2}-\xi^{2}\right)} \cong \mathbf{C} \delta_{k, 0} \delta_{d_{\eta}, l} .
$$

An arbitrary state in the complex $C_{\frac{\infty}{2}}\left(\operatorname{Vir}, \operatorname{Vir}_{0} ; F_{\xi^{\prime}, \eta_{l}} \otimes F_{\xi, \eta}\right)$ is a linear combination of basis states

$$
b_{-n_{1}} \ldots b_{-n_{i}} c_{-m_{1}} \ldots c_{-m_{j}} w_{g h} \otimes j_{-r_{1}} \ldots j_{-r_{z}} v_{\xi^{\prime}, \eta_{l}} \otimes j_{-s_{1}} \ldots j_{-s_{u}} v_{\xi, \eta}
$$

with total energy zero (remember the condition $L_{0}^{\text {tot }}=0$ ):

$$
\begin{aligned}
& -1+\frac{1}{2}\left(\eta_{l}^{2}-\xi^{\prime 2}\right)+\frac{1}{2}\left(\eta^{2}-\xi^{2}\right)+\left(n_{1}+\cdots+n_{i}\right) \\
& +\left(m_{1}+\cdots+m_{j}\right)+\left(r_{1}+\cdots+r_{t}\right)+\left(s_{1}+\cdots+s_{u}\right)=0 .
\end{aligned}
$$

By Eq. (3.67), a $d$-cycle will be a trivial $d$-cycle, unless all the $m, n, r, s$, are zero, and

$$
l=d_{\eta} \equiv N .
$$

This means that among all the $d$-cycles in any of the spaces

$$
\left\{C_{\frac{\infty}{2}+k}\left(\text { Vir, } \operatorname{Vir}_{0} ; F_{\xi^{\prime}, \eta_{l}} \otimes F_{\xi, \eta}\right)\right\}_{(k, l) \in \mathbf{Z} \times \mathbf{Z}}
$$

there is a single non-trivial $d$-cycle:

$$
w_{0}=w_{g h} \otimes v_{\xi^{\prime}, \eta_{N}} \otimes v_{\xi, \eta}
$$

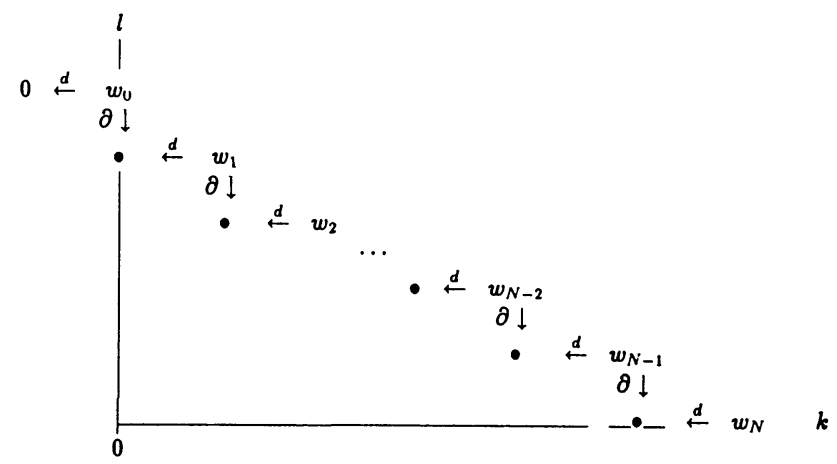

Fig. 5. Zhe zig-zag method 
which occupies the $(0, N)^{\text {th }}$ space. Let's put the spaces (3.71) in an integral lattice on the $(k, l)$-plane. Each integral point $(k, l)$ is occupied by the $(k, l)^{\text {th }}$ space $C_{\frac{\infty}{2}+k}\left(\mathrm{Vir}, \mathrm{Vir}_{0} ; F_{\xi^{\prime}, \eta_{l}} \otimes F_{\xi, \eta}\right)$. The maps that intertwine two adjacent spaces are the (horizontal) BRST differential $d$, and the (vertical) Felder differential $\partial$ [induced by Felder's map $\delta$ in (3.17)]. By construction, these two differentials anti-commute:

$$
\{d, \partial\}=0 \text {. }
$$

Assuming that $N>0$ (the case $N=0$ is trivial), we now begin the zig-zag walk on the plane, starting from $(0, N)$. First

$$
d \partial w_{0}=-\partial d w_{0}=0
$$

i.e. $\partial w_{0}$ is a $d$-cycle in the $(0, N-1)^{\text {th }}$ space. By (3.72), it must be trivial, i.e.

$$
\partial w_{0}=d w_{1} \quad \text { for some } w_{1} \text {, at }(1, N-1) .
$$

Once again $\partial w$ is at $(1, N-2)$, and

$$
d \partial w_{1}=-\partial d w_{1}=-\partial^{2} w_{0}=0
$$

implies that

$$
\partial w_{1}=d w_{2} \text { for some } w_{2} \text { at }(2, N-2) .
$$

Continuing this way, we end up with

$$
\partial w_{N-1}=d w_{N} \text { for some } w_{N} \text { at }(N, 0) .
$$

Clearly $d w_{N-1} \in \operatorname{Ker} \partial_{0}$, where $\partial_{0}$ is $\partial$ restricted to the spaces in the zeroth row of Fig. 5. We claim that $w_{N}$ can also be chosen to be in $\operatorname{Ker} \partial_{0}$. By definition, this $\partial$ is induced by Felder's map

$$
\delta_{0}: F_{\xi^{\prime}, \eta_{0}} \rightarrow F_{\xi^{\prime}, \eta_{-1}}
$$

This means that

$$
\operatorname{Ker} \partial_{0}=C_{\frac{\infty}{2}+N-1}\left(\operatorname{Vir}, \operatorname{Vir}_{0} ; \operatorname{Ker} \delta_{0} \otimes F_{\xi, \eta}\right) .
$$

Now the truncated Felder resolution

$$
0 \rightarrow \operatorname{Ker} \delta_{0} \hookrightarrow F_{\xi^{\prime}, \eta_{0}} \stackrel{\delta_{0}}{\rightarrow} F_{\xi^{\prime}, \eta_{-1}} \rightarrow \cdots
$$

is exact, by construction. But we already know that each $F_{\xi^{\prime}, \eta_{l}}$ in this sequence has no BRST homology because $l=0,-1, \ldots$ all differ from $N \stackrel{\text { }}{=} d_{\eta}$ [Eq. (3.70)]. This means that $\operatorname{Ker} \delta_{0}$, in the exact sequence above, cannot have any BRST homology either, i.e. any $d$-cycle in the BRST complex (3.80) is trivial. In particular,

$$
d \partial w_{N-1}=-\partial d w_{N-1}=0 \Rightarrow \partial w_{N-1}=d w_{N} \quad \text { for some } w_{N} \in \operatorname{Ker} \partial_{0} .
$$

To summarize: we have obtained a sequence of states $w_{0}, w_{1}, \ldots, w_{N}$ with the properties:

$$
\begin{gathered}
w_{i} \text { is in }(i, N-i)^{\mathrm{th}} \text { space } \\
\partial w_{i}=d w_{i+1} \quad i<N \\
\partial w_{N}=0
\end{gathered}
$$


Theorem 3.4. For $i \xi=\frac{p+q}{\sqrt{2 p q}}$, $i \eta=\frac{|2 p q t+p r+\varepsilon q s|}{\sqrt{2 p q}}$, the homology space $H_{\frac{\infty}{2}}+d_{\eta}$ $\left(\operatorname{Vir}, \operatorname{Vir}_{0} ; L\left(c_{p, q}, \Delta_{r, s}\right) \otimes F_{\xi, \eta}\right)$ has a representative of the form

$$
\bar{w}_{N}=\left[\left(d^{-1} \partial\right)^{N} w_{0}+\operatorname{Im} \partial\right] \in \operatorname{Ker} \partial / \operatorname{Im} \partial .
$$

The $\frac{\infty}{2}$-homology class of $\bar{w}_{N}$ is independent of the choices of inverse images under $d$.

Proof. First, $\bar{w}_{N}$ is a $d$-cycle (BRST invariant):

$$
d \bar{w}_{N}=\partial\left(d^{-1} \partial\right)^{N-1} w_{0}+\operatorname{Im} \partial \equiv 0(\bmod \operatorname{Im} \partial) .
$$

Suppose $\bar{w}_{N}$ is trivial, i.e.

$$
\bar{w}_{N}=-d \bar{u}_{N}, \quad \text { for some } u_{N} \in \operatorname{Ker} \partial \text { at }(N+1,0) .
$$

Then

$$
w_{N}+d u_{N}=\partial u_{N-1}, \quad \text { for some } u_{N-1} \text { at }(N, 1) \Rightarrow d w_{N}=-\partial u_{N-1} .
$$

Combining (3.84) (for $i=N-1$ ) and (3.88) gives

$$
w_{N-1}+d u_{N-1} \in \operatorname{Ker} \partial \text { at }(N-1,1) .
$$

By exactness of Felder's resolution (away from dimension zero), we have

$$
w_{N-1}+d u_{N-1}=\partial u_{N-1}, \quad \text { for some } u_{N-2} \text { at }(N-1,2) .
$$

Continuing this way, we get

$$
w_{1}+d u_{1}=\partial u_{0}, \quad \text { for some } u_{0} \text { at }(1, N) .
$$

Now $w_{0}$ is the lowest energy state in the space $C_{\frac{\infty}{2}}\left(\operatorname{Vir}, \operatorname{Vir}_{0} ; F_{\xi^{\prime}, \eta_{N}} \otimes F_{\xi, \eta}\right)$ with $L_{0}^{\text {tot }} w_{0}=0, g h \# w_{0}=0$. So there is no other independent state $w$ in this space, since $w$ would have higher energy, implying that $L_{0}^{\text {tot }} w \neq 0$. But by (3.91), $u_{0}$ is in this space and has $g h \# u_{0}=-1$. Thus $u_{0}$ must be zero. Since $\partial w_{0}=d w_{1}$, Eq. (3.91) means that

$$
\partial w_{0}=d w_{1}=-d^{2} u_{1}=0 .
$$

By exactness of Felder's resolution and Eq. (3.92),

$$
\begin{aligned}
w_{0} & =w_{g h} \otimes v_{\xi^{\prime}, \eta_{N}} \otimes v_{\xi, \eta} \\
& =\partial\left(w_{g h} \otimes v \otimes v_{\xi, \eta}\right) \\
& =w_{g h} \otimes \delta_{N+1} v \otimes v_{\xi, \eta}
\end{aligned}
$$

for some $v \in F_{\xi^{\prime}, \eta_{N+1}}$, having the same energy, $\frac{1}{2}\left(\eta_{N}^{2}-\xi^{2}\right)$, as $v_{\xi^{\prime}, \eta_{N}}$. But this is impossible because the lowest energy in $F_{\xi^{\prime}, \eta_{N+1}}$ is $\frac{1}{2}\left(\eta_{N+1}^{2}-\xi^{\prime 2}\right)$ and yet

$$
\frac{1}{2}\left(\eta_{N+1}^{2}-\xi^{\prime 2}\right)>\frac{1}{2}\left(\eta_{N}^{2}-\xi^{2}\right) .
$$

This contradiction shows that the supposition (3.87) is false. Thus $\bar{w}_{N}$ is indeed a non-trivial $d$-cycle.

We now show the last statement of Theorem 3.4. Suppose that $w_{0}, w_{1}^{\prime}, \ldots, w_{N}^{\prime}$ is another sequence satisfying (3.83)-(3.85). Then as before,

$$
\partial w_{0}=d w_{1}=d w_{1}^{\prime} \Rightarrow w_{1}-w_{1}^{\prime}=d w_{1}^{\prime \prime} \text { for some } w_{1}^{\prime \prime} \text {. }
$$


This gives

$$
\begin{aligned}
\partial w_{1}^{\prime}-\partial w_{1} & =-d \partial w_{1}^{\prime \prime}=d w_{2}^{\prime}-d w_{2} \\
& \Rightarrow w_{2}^{\prime}-w_{2}+\partial w_{1}^{\prime \prime} \\
& =d w_{2}^{\prime \prime} \\
& \quad \text { for some } w_{2}^{\prime \prime} .
\end{aligned}
$$

Once again, we get

$$
\partial w_{2}^{\prime}-\partial w_{2}=-d \partial w_{2}^{\prime \prime} .
$$

Continuing this way, we end up with

$$
w_{N}^{\prime}-w_{N}+\partial w_{N-1}^{\prime \prime}=d w_{N}^{\prime \prime}
$$

Projecting onto the quotient space

$$
\begin{aligned}
& \frac{\operatorname{Ker}\left[\partial: C_{\frac{\infty}{2}+N}\left(\operatorname{Vir}, \operatorname{Vir}_{0} ; F_{\xi^{\prime}, \eta_{0}} \otimes F_{\xi, \eta}\right) \rightarrow C_{\frac{\infty}{2}+N}\left(\operatorname{Vir}, \operatorname{Vir}_{0} ; F_{\xi^{\prime}, \eta_{-1}} \otimes F_{\xi, \eta}\right)\right]}{\operatorname{Im}\left[\partial: C_{\frac{\infty}{2}+N}\left(\operatorname{Vir}_{V^{\prime}} \operatorname{Vir}_{0} ; F_{\xi^{\prime}, \eta_{1}} \otimes F_{\xi, \eta}\right) \rightarrow C_{\frac{\infty}{2}+N}\left(\operatorname{Vir}_{\operatorname{Vir}_{0}} ; F_{\xi^{\prime}, \eta_{0}} \otimes F_{\xi, \eta}\right)\right]} \\
& \quad=C_{\frac{\infty}{2}+N}\left(\operatorname{Vir}, \operatorname{Vir}_{0} ; L\left(c_{p, q}, \Delta_{r, s}\right) \otimes F_{\xi, \eta}\right)
\end{aligned}
$$

we get

$$
\bar{w}_{N}^{\prime}-\bar{w}_{N}=d \bar{w}_{N}^{\prime \prime} .
$$

It follows that $\bar{w}_{N}^{\prime}, \bar{w}_{N}$ are two equivalent $d$-cycles in $C_{\frac{\infty}{2}+N}\left(\operatorname{Vir}, \operatorname{Vir}_{0} ; L\left(c_{p, q}, \Delta_{r, s}\right) \otimes\right.$ $\left.F_{\xi, \eta}\right)$. This completes the proof.

\subsection{Computing $H_{\frac{\infty}{2}+*}\left(\operatorname{Vir}, \mathbf{C} \hat{c} ; L\left(c_{p, q}, \Delta_{r, s}\right) \otimes \mathbf{F}_{\xi, \eta}\right)$}

In Sect. 1, we formulated our problems in terms of the relative $\frac{\infty}{2}$-complex

$$
\begin{aligned}
& C_{\frac{\infty}{2}+*}\left(\operatorname{Vir}, \operatorname{Vir}_{0} ; L\left(c_{p, q}, \Delta_{r, s}\right) \otimes F_{\xi, \eta}\right) \\
& \quad \stackrel{\text { def }}{=}\left\{w \in L\left(c_{p, q}, \Delta_{r, s}\right) \otimes F_{\xi, \eta} \otimes \wedge_{\frac{\infty}{2}+*}(\operatorname{Vir} / \mathbf{C} \hat{c}) \mid L_{0}^{\text {tot }} w=0=b_{0} w\right\} .
\end{aligned}
$$

We now examine the full complex

$$
\begin{aligned}
& C_{\frac{\infty}{2}+*}\left(\operatorname{Vir}, \mathbf{C} \hat{c} ; L\left(c_{p, q}, \Delta_{r, s}\right) \otimes F_{\xi, \eta}\right) \\
& \quad \stackrel{\text { def }}{=} L\left(c_{p, q}, \Delta_{r, s}\right) \otimes F_{\xi, \eta} \otimes \wedge_{\frac{\infty}{2}+*}(\operatorname{Vir} / \mathbf{C} \hat{c}) .
\end{aligned}
$$

As before, we need only to consider the case when the total central charge is zero

$$
1-12 \xi^{2}+c_{p, q}-26=0 .
$$

Since $L_{0}^{\text {tot }}$ is BRST exact,

$$
\left\{d, b_{0}\right\}=L_{0}^{\text {tot }}
$$

any $d$-cycle $w$ with non-zero $L_{0}^{\text {tot }}$ eigenvalue $\lambda$, is trivial:

$$
\lambda w=L_{0}^{\mathrm{tot}} w=\left(d b_{0}+b_{0} d\right) w=d b_{0} w \Rightarrow w=\frac{1}{\lambda} d b_{0} w .
$$


Thus

$$
\begin{aligned}
& H_{\frac{\infty}{2}+*}\left(\operatorname{Vir}, \mathbf{C} \hat{c} ; L\left(c_{p, q}, \Delta_{r, s}\right) \otimes F_{\xi, \eta}\right) \\
& \quad \cong H_{*}\left(C_{\frac{\infty}{2}+*}\left(\operatorname{Vir}, \mathbf{C} \hat{c} ; L\left(c_{p, q}, \Delta_{r, s}\right) \otimes F_{\xi, \eta}\right)^{L_{0}^{\mathrm{tot}}}, d\right) .
\end{aligned}
$$

Using $b_{0}^{2}=0$ and definitions (3.101), (3.102), we can easily show that

$$
\begin{aligned}
0 & \rightarrow C_{\frac{\infty}{2}+*}\left(\operatorname{Vir}, \operatorname{Vir}_{0} ; L\left(c_{p, q}, \Delta_{r, s}\right) \otimes F_{\xi, \eta}\right) \\
& \hookrightarrow C_{\frac{\infty}{2}+*}\left(\operatorname{Vir}, \operatorname{C} \hat{c} ; L\left(c_{p, q}, \Delta_{r, s}\right) \otimes F_{\xi, \eta}\right)^{L_{0}^{\text {tot }}} \\
& \stackrel{(-1)^{*} b_{0}}{\rightarrow} C_{\frac{\infty}{2}+*+1}\left(\operatorname{Vir}, \operatorname{Vir}_{0} ; L\left(c_{p, q}, \Delta_{r, s}\right) \otimes F_{\xi, \eta}\right) \rightarrow 0
\end{aligned}
$$

is an exact sequence of complexes. Thus we have a long exact sequence

$$
\begin{aligned}
\cdots & \rightarrow H_{\frac{\infty}{2}+n}\left(\operatorname{Vir}, \operatorname{Vir}_{0} ; L\left(c_{p, q}, \Delta_{r, s}\right) \otimes F_{\xi, \eta}\right) \\
& \rightarrow H_{\frac{\infty}{2}+n}\left(\operatorname{Vir}, \mathbf{C} \hat{c} ; L\left(c_{p, q}, \Delta_{r, s}\right) \otimes F_{\xi, \eta}\right) \\
& \rightarrow H_{\frac{\infty}{2}+n+1}\left(\operatorname{Vir}, \operatorname{Vir}_{0} ; L\left(c_{p, q}, \Delta_{r, s}\right) \otimes F_{\xi, \eta}\right) \\
& \rightarrow H_{\frac{\infty}{2}+n-1}\left(\operatorname{Vir}, \operatorname{Vir}_{0} ; L\left(c_{p, q}, \Delta_{r, s}\right) \otimes F_{\xi, \eta}\right) \rightarrow \cdots .
\end{aligned}
$$

Note that we have used Eq. (3.106) to get rid of $H_{*}\left(C_{\frac{\infty}{2}+*}\left(\operatorname{Vir}, \mathbf{C} \hat{c} ; L\left(c_{p, q}, \Delta_{r, s}\right) \otimes\right.\right.$

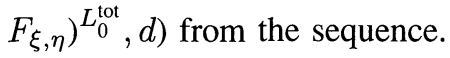

Theorem 3.5. For $\sqrt{-1} \xi=\frac{p+q}{\sqrt{2 p q}}$,

(a) $H_{\frac{\infty}{2}}\left(\operatorname{Vir}, \mathbf{C} \hat{c} ; L\left(c_{p, q}, \Delta_{r, s}\right) \otimes F_{\xi, \eta}\right) \neq 0$ iff $\sqrt{-1} \eta=\sigma \frac{2 p q t+p r+\varepsilon q s}{\sqrt{2 p q}}, t \in \mathbf{Z}$, $\sigma, \varepsilon= \pm 1$.

(b) For the special values of $(\xi, \eta)$ given above,

$$
H_{\frac{\infty}{2}+n}\left(\operatorname{Vir}, \mathbf{C} \hat{c} ; L\left(c_{p, q}, \Delta_{r, s}\right) \otimes F_{\xi, \eta}\right) \cong \delta_{n+\pi(\eta) d_{\eta}, 0} \mathbf{C} \oplus \delta_{n+\pi(\eta) d_{\eta},-1} \mathbf{C} .
$$

Proof. For fixed $(\xi, \eta)$, it can be easily shown that the sequence (3.108) terminates on both ends. If

$$
\sqrt{-1} \eta \neq \sigma \frac{2 p q t+p r+\varepsilon q s}{\sqrt{2 p q}}, t \in \mathbf{Z}, \quad \sigma, \varepsilon= \pm 1
$$

then by Theorem 3.1, we have

$$
H_{\frac{\infty}{2}+*}\left(\operatorname{Vir}, \operatorname{Vir}_{0} ; L\left(c_{p, q}, \Delta_{r, s}\right) \otimes F_{\xi, \eta}\right)=0 .
$$

By exactness of (3.108), we get

$$
H_{\frac{\infty}{2}+*}\left(\operatorname{Vir}, \mathbf{C} \hat{c} ; L\left(c_{p, q}, \Delta_{r, s}\right) \otimes F_{\xi, \eta}\right)=0 .
$$

Conversely, if

$$
\sqrt{-1} \eta=\sigma \frac{2 p q t+p r+\varepsilon q s}{\sqrt{2 p q}}, t \in \mathbf{Z}, \quad \sigma, \varepsilon= \pm 1,
$$

then letting $k=-\pi(\eta) \dot{d}_{\eta}$, we get from Theorem 3.1,

$$
H_{\frac{\infty}{2}+n}\left(\operatorname{Vir}, \operatorname{Vir}_{0} ; L\left(c_{p, q}, \Delta_{r, s}\right) \otimes F_{\xi, \eta}\right) \cong \delta_{n, k} \mathbf{C} .
$$


Substituting this into (3.108), we get

$$
\begin{aligned}
& 0 \rightarrow H_{\frac{\infty}{2}+n}\left(\operatorname{Vir}, \mathbf{C} \hat{c} ; L\left(c_{p, q}, \Delta_{r, s}\right) \otimes F_{\xi, \eta}\right) \rightarrow 0 \text { for } n \neq k, k-1, \\
& 0 \rightarrow \mathbf{C} \rightarrow H_{\frac{\infty}{2}+k}\left(\operatorname{Vir}, \mathbf{C} \hat{c} ; L\left(c_{p, q}, \Delta_{r, s}\right) \otimes F_{\xi, \eta}\right) \rightarrow 0 \\
& 0 \rightarrow H_{\frac{\infty}{2}+k-1}\left(\operatorname{Vir}, \mathbf{C} \hat{c} ; L\left(c_{p, q}, \Delta_{r, s}\right) \otimes F_{\xi, \eta}\right) \rightarrow \mathbf{C} \rightarrow 0 .
\end{aligned}
$$

This proves part (b) and the second half of part (a).

\subsection{Discussions}

A discussion of gravity would be incomplete without mentioning supergravity [13]. As anticipated, the formulation of the problems studied in $[33,34]$ and this paper has natural super extensions. To make our discussion more focused, let's restrict ourselves to the $c<1$ case.

The quantum state space of 2D supergravity has the same form as (1.3). The gravitational sector is now given by the super Liouville theory with the Neveu-Schwarz algebra $\mathrm{Vir}^{\mathrm{NS}}$, or the Ramond algebra $\mathrm{Vir}^{\mathrm{R}}$, being the underlying symmetry. This symmetry is generated by the stress energy $T$ and its superpartner $G$ :

$$
\begin{gathered}
T=-\frac{1}{2}(\partial \varphi)^{2}+i \xi \partial \varphi+\frac{1}{2} \partial \psi \psi+\frac{\chi}{8} z^{-2} \\
G=i \psi \partial \varphi+2 \xi \partial \psi
\end{gathered}
$$

where $\chi=0\left(\chi=\frac{1}{2}\right)$ gives the Neveu-Schwarz (Ramond) algebra. Here $\varphi$ is the spin 0 Liouville field and $\psi$ is its spin $\frac{1}{2}$ superpartner. Equations (3.115), (3.116) define a super extension of the Feigin-Fuchs module.

We will restrict ourselves to the case in which the matter sector is one of the super minimal models. The $(p, q)$ model has central charge

$$
c_{p, q}=1-\frac{2(p-q)^{2}}{p q}
$$

where either $p, q \in 2 \mathbf{N}-1, \operatorname{gcd}(p, q)=1$ or $p, q \in 2 \mathbf{N}, \frac{p-q}{2} \in 2 \mathbf{N}-1, \operatorname{gcd}\left(\frac{p}{2}, \frac{q}{2}\right)=$ 1 (we follow the conventions of [40]). The primary fields $\Phi_{r, s}$ in this model have conformal dimensions

$$
\begin{gathered}
\Delta_{r, s}=\frac{(p r-q s)^{2}-(p-q)^{2}}{8 p q}+\frac{\chi}{8}, \\
1 \leq r \leq q-1, \quad 1 \leq s \leq p-1, \quad r-s \in 2 \chi+2 \mathbf{Z} .
\end{gathered}
$$

The representation spaces of the model are the irreducible modules $L\left(c_{p, q}, \Delta_{r, s}\right)$ over $\mathrm{Vir}^{\mathrm{NS}}$ or $\mathrm{Vir}^{\mathrm{R}}$. (Note that the notations we use here are the same as those used in the bosonic case.) Finally, the ghost sector is generated by the (super) conformal fields $(b, c),(\beta, \gamma)$ of dimensions $(2,-1),\left(\frac{3}{2},-\frac{1}{2}\right)$ respectively.

Now the super versions of Problems 1 and 2 (Sect. 1) can be easily formulated by replacing Vir by $\operatorname{Vir}^{\mathrm{NS}}$ (or $\mathrm{Vir}^{\mathrm{R}}$ ), the minimal model representations and FeiginFuchs modules by their respective super extensions, as discussed above. Recall that in Sects. 2 and 3, we relied heavily on the embedding structure of Verma modules over Vir, in order to solve Problem 1. To our knowledge, the full embedding structure of Verma modules over $\mathrm{Vir}^{\mathrm{NS}}$ (or $\mathrm{Vir}^{\mathrm{R}}$ ) has not been worked out (see [40]). But one 
should anticipate that the super versions of Theorem 2.1 and 2.2 will hold. Actually, one can answer at least half of Problem 1 in the super case without knowing the full embedding structure. Namely (cf. Theorem 3.1) if

$$
\begin{aligned}
\xi & =i \frac{p+q}{\sqrt{4 p q}}, \\
\eta & =i \varepsilon_{1} \frac{\left(2 p q t+p r+\varepsilon_{2} q s\right)}{\sqrt{4 p q}}, t \in \mathbf{Z}, \quad \varepsilon_{1}, \varepsilon_{2}= \pm 1
\end{aligned}
$$

then

$$
H_{\frac{\infty}{2}}\left(\operatorname{Vir}^{\mathrm{Ns}}, \operatorname{Vir}_{0}^{\mathrm{Ns}} ; L\left(c_{p, q}, \Delta_{r, s}\right) \otimes F_{\xi, \eta}\right) \neq 0,
$$

A similar statement holds for $\operatorname{Vir}^{\mathrm{R}}$. The proof requires the use of Rocha's character formulas [40] for $L\left(c_{p, q}, \Delta_{r, s}\right)$. Problem 2 in the super case also requires some further work. One should first construct Felder's resolution for the super minimal model representations $L\left(c_{p, q}, \Delta_{r, s}\right)$. One can then apply our rotation trick to compute the BRST homology exactly just as we did in the bosonic case (Sect. 3).

We can also compute the scaling dimensions of those physical states (3.121) corresponding to the super Liouville charges (3.12). Imitating the arguments in [33], we obtain a gravitational dressing equation for each non-trivial BRST invariant state:

$$
\Delta-\frac{\chi}{8}-\frac{1}{2} \alpha(\alpha-Q)=\frac{1}{2}
$$

where $\alpha=i(\xi-\eta), \Delta \in\left\{a_{t}, b_{t}\right\}_{t \in \mathbf{Z}}$,

$$
\begin{aligned}
& a_{t}=\frac{(2 p q t+p r+q s)^{2}-(p-q)^{2}}{8 p q}+\frac{\chi}{8}, \\
& b_{t}=\frac{(2 p q t+p r-q s)^{2}-(p-q)^{2}}{8 p q}+\frac{\chi}{8} .
\end{aligned}
$$

When $\Delta=b_{0}$, Eq. (3.122) reduces to the one obtained by [13]. From (3.122) and applying Seiberg's condition, we obtain the dressed dimensions

$$
\hat{\Delta}=1-\frac{\alpha}{\alpha_{\text {low }}}=\frac{|2 p q t+p r+\varepsilon q s|-m_{p, q}}{p+q-m_{p, q}},
$$

where $t \in \mathbf{Z}, \varepsilon= \pm 1$ and $(r, s)$ range over (3.119). Here $m_{p, q}$ is the minimum value of $|p r-q s|$ as $(r, s)$ range over (3.119).

This completes our discussion of supergravity in the conformal gauge. We now turn our discussion to a different problem.

In Sect. 3, a key step for computing $H_{\frac{\infty}{2}+*}\left(\operatorname{Vir}, \operatorname{Vir}_{0} ; F_{\xi, \eta} \otimes L\left(c_{p, q}, \Delta_{r, s}\right)\right)$ was to consider (Eq. (3.26))

$$
H_{\frac{\infty}{2}+*}\left(\operatorname{Vir}, \operatorname{Vir}_{0} ; F_{\xi, \eta} \otimes F_{\xi^{\prime}, \eta^{\prime}}\right)=?,
$$

where $\xi^{\prime}, \eta^{\prime}, \xi, \eta$ satisfy certain conditions. In our earlier work on $c=1$ gravity, we also encountered the same problem (3.125). This problem can be generalized mathematically to the case when $\xi^{\prime}, \eta^{\prime}, \xi, \eta$ are completely arbitrary. The solution to this problem may eventually be useful for studying $c>1$ matter coupled to gravity. Thus it is interesting to work out $(3.125)$ in complete generality. It turns out that our rotation trick (Proposition 3.2, Sect. 3; Proposition 2.1 [34]) works equally well in the general case. Specifically, the BRST homology is completely determined by values 
of the two fundamental $S O(2, \mathrm{C})$-invariants: the symmetric and the anti-symmetric 2-forms.

Proposition 3.6. Let $\xi^{\prime}, \eta^{\prime}, \xi, \eta \in \mathbf{C}$. (a) If $\xi^{2}+\xi^{\prime 2} \neq-2$, then

$$
H_{\frac{\infty}{2}+n}\left(\operatorname{Vir}, \operatorname{Vir}_{0} ; F_{\xi, \eta} \otimes F_{\xi^{\prime}, \eta^{\prime}}\right)=0
$$

for all $n$.

(b) If $\xi^{2}+\xi^{\prime 2}=-2$, then

$$
\begin{aligned}
& H_{\frac{\infty}{2}+n}\left(\operatorname{Vir}, \operatorname{Vir}_{0} ; F_{\xi, \eta} \otimes F_{\xi^{\prime}, \eta^{\prime}}\right) \\
& \quad \cong H_{\frac{\infty}{2}+n}\left(\operatorname{Vir}, \operatorname{Vir}_{0} ; F_{-i \sqrt{2}, i \sqrt{2}\left(\xi \eta+\xi^{\prime} \eta^{\prime}\right) / 2} \otimes F_{0, i \sqrt{2}\left(\xi \eta^{\prime}-\xi^{\prime} \eta\right) / 2}\right)
\end{aligned}
$$

for all $n$.

Note that in case (b), the right-hand side of (3.127) has already been calculated explicitly (see Corollary 2.3 and Theorem 2.5 in [34]).

\section{Appendix A. Resolutions}

In this appendix, we illustrate how to compute semi-infinite homology using resolutions and spectral sequences. Throughout this appendix, let $\mathscr{K}$ be a Z-graded Lie algebra, $\mathscr{b} \subset \mathscr{K}_{0}$ be a subalgebra which acts semi-simply on $\mathscr{K}$ via the adjoint action, and $V_{1}, V, V^{\prime}, V^{\prime \prime}$ be $\mathbf{Z}$-graded $\mathscr{K}$-modules in which $\mathscr{A}$ acts semi-simply. Moreover, assume each $\mathscr{K}_{n}, V_{n}$ etc. is finite dimensional.

Proposition A.1. The functor $C_{\frac{\infty}{2}+*}\left(\mathscr{R}, \mathscr{A} ; V_{1} \otimes-\right)$ is exact. That is, if

$$
0 \rightarrow V^{\prime} \rightarrow V \rightarrow V^{\prime \prime} \rightarrow 0
$$

is exact, then

$$
\begin{aligned}
0 \rightarrow C_{\frac{\infty}{2}+*}\left(\mathscr{K}, \mathscr{b} ; V_{1} \otimes V^{\prime}\right) & \rightarrow C_{\frac{\infty}{2}+*}\left(\mathscr{K}, \mathscr{A}, V_{1} \otimes V^{\prime}\right) \\
& \rightarrow C_{\frac{\infty}{2}+*}\left(\mathscr{K}, \mathscr{b} ; V_{1} \otimes V^{\prime \prime}\right) \rightarrow 0
\end{aligned}
$$

is an exact sequence of $\frac{\infty}{2}$-chain complexes.

Proof. Recall that

$$
C_{\frac{\infty}{2}+*}\left(\mathscr{K}, \mathscr{b} ; V_{1} \otimes-\right)=\left(-\otimes V_{1} \otimes \wedge_{\frac{\infty}{2}+*} \mathscr{K}^{L(\mathscr{C})}\right)^{\mathscr{b}},
$$

where $\wedge_{\frac{\infty}{2}+*} \mathscr{K}^{\iota(\mathscr{C})}$ denotes the subspace of $\wedge_{\frac{\infty}{2}+*} \mathscr{K}$ annihilated by all $\iota(x), x \in \mathscr{C}$. Now

$$
\begin{aligned}
0 \rightarrow V^{\prime} \otimes V_{1} \otimes \wedge_{\frac{\infty}{2}+*} \mathscr{K}^{\iota(, / b)} & \rightarrow V \otimes V_{1} \otimes \wedge_{\frac{\infty}{2}+*} \mathscr{K}^{\iota(, \mathscr{l})} \\
& \rightarrow V^{\prime \prime} \otimes V_{1} \otimes \wedge_{\frac{\infty}{2}+*} \mathscr{K}^{\iota(\mathscr{b})} \rightarrow 0
\end{aligned}
$$

is clearly exact as a sequence $\mathscr{A}$-modules. Now by assumption, each term in (A.2) is semi-simple. This immediately implies that the sequence of $\mathscr{C}$-invariants

$$
\begin{aligned}
0 \rightarrow\left\{V^{\prime} \otimes V_{1} \otimes \wedge_{\frac{\infty}{2}+*} \mathscr{K}^{\iota(\mathscr{C})}\right\}^{\mathscr{\ell}} & \rightarrow\left\{V \otimes V_{1} \otimes \wedge_{\frac{\infty}{2}+*} \mathscr{K}^{\iota(. \mathscr{C})}\right\}^{\mathscr{\ell}} \\
& \rightarrow\left\{V^{\prime \prime} \otimes V_{1} \otimes \wedge_{\frac{\infty}{2}+*} \mathscr{K}^{\iota(\mathscr{C})}\right\}^{\mathscr{\ell}} \rightarrow 0
\end{aligned}
$$

is also exact. 
Let $N$ be a $\mathscr{K}$-module. An exact sequence of $\mathscr{K}$-modules:

$$
\cdots \rightarrow M_{1} \stackrel{\phi_{1}}{\rightarrow} M_{0} \stackrel{\phi_{0}}{\rightarrow} N \rightarrow 0
$$

is called a resolution of $N$. In this case, one has

$$
\begin{aligned}
\operatorname{Ker} \phi_{i-1} & =\operatorname{Im} \phi_{i} \quad i \geq 0, \\
N & \cong M_{0} / \operatorname{Im} \phi_{1} .
\end{aligned}
$$

Thus the truncated sequence

$$
\cdots \rightarrow M_{1} \stackrel{\phi_{0}}{\rightarrow} M_{0} \rightarrow 0
$$

is also exact except at the zeroth dimension. By (A.5), (A.6) this sequence is a chain complex $\left\{M_{*}, \phi_{*}\right\}$ with homology

$$
H_{n}\left(M_{*}, \phi_{*}\right) \cong \delta_{n, 0} N \text {. }
$$

By abuse of language, we will also call the sequence (A.7) a resolution of $N$. More generally, given a sequence of $\mathscr{K}$-modules

$$
\cdots \rightarrow M_{1} \stackrel{\phi_{1}}{\rightarrow} M_{0} \stackrel{\phi_{0}}{\rightarrow} M_{-1} \rightarrow \cdots
$$

with

$$
\begin{aligned}
\operatorname{Ker} \phi_{i} & =\operatorname{Im} \phi_{i+1}, \quad i \neq 0, \\
\operatorname{Im} \phi_{1} & \subseteq \operatorname{Ker} \phi_{0}, \\
N & \cong \operatorname{Ker} \phi_{0} / \operatorname{Im} \phi_{1},
\end{aligned}
$$

we called $\left\{M_{*}, \phi_{*}\right\}$ a (2-sided) resolution of the $\mathscr{K}$-module $N$. Our goal is to make use of this extra structure of $N$, to study the $\frac{\infty}{2}$-homology

$$
H_{\frac{\infty}{2}+*}\left(\mathscr{K}, \mathscr{A} ; V_{1} \otimes N\right),
$$

By Proposition A.1, the functor $C_{\frac{\infty}{2}+*}\left(\mathscr{R}, \mathscr{A} ; V_{1} \otimes-\right)$ preserves exact sequences. Let's abbreviate it by

$$
C_{\frac{\infty}{2}+*}-=C_{\frac{\infty}{2}+*}\left(\mathscr{K}, \mathscr{b} ; V_{1} \otimes-\right) .
$$

Thus given a resolution (A.9) of $N$, we get a sequence of vector spaces for each $r$,

$$
\cdots \rightarrow C_{\frac{\infty}{2}+r} M_{1} \stackrel{\phi_{1}^{\prime}}{\rightarrow} C_{\frac{\infty}{2}+r} M_{0} \stackrel{\phi_{0}^{\prime}}{\rightarrow} C_{\frac{\infty}{2}+r} M_{-1} \rightarrow \cdots
$$

which is exact except at the zeroth dimension. It clearly remains so if we replace the induced maps $\phi_{i}^{\prime}$ by

$$
\partial \stackrel{\text { def }}{=}(-1)^{r} \phi_{i}^{\prime}
$$

Since the $\phi_{i}^{\prime}$ are induced by the module maps $\phi_{i}$, they commute with the $\frac{\infty}{2}$ differential $d$. Thus by (A.16), we get an anti-commutative diagram

$$
\begin{array}{cc}
C_{\frac{\infty}{2}+r} M_{s} \stackrel{d}{\longrightarrow} C_{\frac{\infty}{2} r-1} M_{s} \\
\quad \downarrow \\
C_{\frac{\infty}{2}+r} M_{s-1} \stackrel{d}{\longrightarrow} C_{\frac{\infty}{2}+r-1} M_{s-1}
\end{array}
$$


This means that $\left\{C_{\frac{\infty}{2}+*} M_{*}, d+\partial\right\}$ is a double complex. Let's calculate the "vertical" homology of (A.17), $H_{*}\left(C_{\frac{\infty}{2}+r} M_{*}, \partial\right)$. But this is just the homology of (A.15):

$$
H_{n}\left(C_{\frac{\infty}{2}+r} M_{*}, \partial\right)=\delta_{n, 0} \frac{\operatorname{Ker}\left(\phi_{0}^{\prime}: C_{\frac{\infty}{2}+r} M_{0} \rightarrow C_{\frac{\infty}{2}+r} M_{-1}\right)}{\operatorname{Im}\left(\phi_{0}^{\prime}: C_{\frac{\infty}{2}+r} M_{1} \rightarrow C_{\frac{\infty}{2}+r} M_{0}\right)} .
$$

Once again, by the exactness of $C_{\frac{\infty}{2}+*}-$, we have

$$
\begin{gathered}
\operatorname{Ker}\left(\phi_{0}^{\prime}: C_{\frac{\infty}{2}+r} M_{0} \rightarrow C_{\frac{\infty}{2}+r} M_{-1}\right) \cong C_{\frac{\infty}{2}+r} \operatorname{Ker} \phi_{0}, \\
\operatorname{Im}\left(\phi_{1}^{\prime}: C_{\frac{\infty}{2}+r} M_{1} \rightarrow C_{\frac{\infty}{2}+r} M_{0}\right) \cong C_{\frac{\infty}{2}+r} \operatorname{Im} \phi_{1},
\end{gathered}
$$

and

$$
\begin{aligned}
H_{n}\left(C_{\frac{\infty}{2}+r} M_{*}, \partial\right) & \cong \delta_{n, 0} \frac{C_{\frac{\infty}{2}+r} \operatorname{Ker} \phi_{0}}{C_{\frac{\infty}{2}+r} \operatorname{Im} \phi_{1}} \\
& \cong \delta_{n, 0} C_{\frac{\infty}{2}+r}\left(\operatorname{Ker} \phi_{0} / \operatorname{Im} \phi_{1}\right) \\
& \cong \delta_{n, 0} C_{\frac{\infty}{2}+r} N .
\end{aligned}
$$

Now consider the double complex (A.17). Recall that $C_{\frac{\infty}{2}+*} M_{*}$ is a $\mathbf{Z}(\mathrm{deg})$-graded space with $\operatorname{deg} d=\operatorname{deg} \partial=0$. So, we can restrict to a deg-homogeneous subcomplex $\left\{C_{\frac{\infty}{2}+*} M_{*}[m], d+\partial\right\}$. We make the following assumption: for fixed $m$,

$$
C_{\frac{\infty}{2}} M_{s}[m]=0 \text { for all } s \text { sufficiently negative. }
$$

In all of our applications, this assumption will hold. Now associated with the double complex are two standard filtrations:

$$
\begin{aligned}
& { }_{1} F^{p}\left(C_{\frac{\infty}{2}} M[m]\right)_{n}=\bigoplus_{\substack{r+s=n \\
r \leq p}} C_{\frac{\infty}{2}+r} M_{s}[m] \\
& { }_{2} F^{p}\left(C_{\frac{\infty}{2}} M[m]\right)_{n}=\bigoplus_{\substack{r+s=n \\
s \leq p}} C_{\frac{\infty}{2}+r} M_{s}[m] .
\end{aligned}
$$

Using the assumption (A.21) and that we are holding $m$ fixed, one can easily show that both filtrations are finite. Thus [25] we have two spectral sequences $\left({ }_{1} E_{n}[m]\right)_{n \geq 0}$, $\left({ }_{2} E_{n}[m]\right)_{n \geq 0}$ which converge finitely to the graded objects associated with $H_{*}\left(C_{\frac{\infty}{2}} M[m], d+\partial\right)$.

\section{Proposition A.2. Let}

$$
\cdots \rightarrow M_{1} \rightarrow M_{0} \rightarrow M_{-1} \rightarrow \cdots
$$

be a (possibly 2-sided) resolution of $\mathscr{K} 6$-module $N$ satisfying assumption (A.21). Then for each $m$, there is a spectral sequence $\left(E_{n}[m]\right)_{n \geq 0}$ such that

$$
\begin{aligned}
& E_{0}^{p, q}[m]=H_{\frac{\infty}{2}+q-p}\left(\mathscr{K}, \mathscr{f} ; V_{1} \otimes M_{p}\right)[m], \\
& E_{1}^{p, q}[m]=H_{p}\left(H_{\frac{\infty}{2}+q-p}\left(\mathscr{K}, \mathscr{b} ; V_{1} \otimes M_{*}\right), \partial\right)[m],
\end{aligned}
$$

and converges finitely to the graded object associated with $H_{*}\left(C_{\frac{\infty}{2}} M[m], d+\partial\right)$ (induced by the filtration $\left.\left\{{ }_{2} F^{p}\left(C_{\frac{\infty}{2}} M[m]\right)\right\}_{p \in \mathbf{Z}}\right)$. Moreover,

$$
H_{*}\left(C_{\frac{\infty}{2}} M[m], d+\partial\right) \cong H_{\frac{\infty}{2}+*}\left(\mathscr{K}, \mathscr{b} ; V_{1} \otimes N\right)[m] .
$$


Proof. Take $\left(E_{n}[m]\right)_{n \geq 0}$ to be $\left.{ }_{2} E_{n}[m]\right)_{n \geq 0}$ above. Computing the first two terms [25], we get

$$
\begin{aligned}
E_{0}^{p, q} & =H_{q-p}\left(C_{\frac{\infty}{2}+*} M_{p}[m], d\right) \\
& \cong H_{\frac{\infty}{2}+q-p}\left(\mathscr{K}, \mathscr{b} ; V_{1} \otimes M_{p}\right)[m], \\
E_{1}^{p, q} & =H_{p}\left(H_{\frac{\infty}{2}+q-p}\left(\mathscr{K}, \mathscr{b} ; V_{1} \otimes M_{*}\right), \partial\right)[m] .
\end{aligned}
$$

To get the third claim, we use $\left\{{ }_{1} E_{n}[m]\right\}_{n \geq 0}$ :

$$
\begin{aligned}
{ }_{1} E_{0}^{p, q}[m] & =H_{q-p}\left(C_{\frac{\infty}{2}+p} M_{*}[m], \partial\right) \\
& \cong H_{q-p}\left(C_{\frac{\infty}{2}+p} M_{*}, \partial\right)[m] \\
& \cong \delta_{p, q} C_{\frac{\infty}{2}+p} N[m] \\
{ }_{1} E_{1}^{p, q} & \left.=H_{p} H_{q-p}\left(C_{\frac{\infty}{2}+*} M_{*}[m], \partial\right), d\right) \\
& \cong \delta_{q, p} H_{p}\left(C_{\frac{\infty}{2}+*} N[m], d\right) \\
& \cong \delta_{q, p} H_{\frac{\infty}{2}+p}\left(\mathscr{\delta}, \mathscr{b} ; V_{1} \otimes N\right)[m] .
\end{aligned}
$$

This immediately implies that the spectral sequence $\left\{{ }_{1} E_{n}[m]\right\}_{n \geq 0}$ collapses:

$$
{ }_{1} E_{\infty}^{p, q}={ }_{1} E_{1}^{p, q} \cong \delta_{q, p} H_{\frac{\infty}{2}+p}\left(\mathscr{K}, \mathscr{C} ; V_{1} \otimes N\right)[m] .
$$

But this spectral sequence converges finitely to the graded object associated with $H_{p}\left(C_{\frac{\infty}{2}} M[m], d+\partial\right)$. Thus

$$
H_{p}\left(C_{\frac{\infty}{2}} M[m], d+\partial\right) \cong H_{\frac{\infty}{2}+p}\left(\mathscr{K}, \mathscr{b} ; V_{1} \otimes N\right)[m] .
$$

\section{References}

1. Banks, T., Douglas, M., Seiberg, N., Shenker, S.: Microscopic and macroscopic loops in nonperturbative two-dimensional gravity. Phys. Lett. B 238, 279 (1980)

2. Bott, R., Tu, L.: Differential forms in algebraic topology. Berlin, Heidelberg, New York: Springer 1982

3. Belavin, A.A., Polyakov, A.M., Zamolodchikov, A.B.: Infinite conformal symmetry in twodimensional quantum field theory. Nucl. Phys. B 241, 333 (1984)

4. Braaten, E., Curtright, T., Thorn, C.: An exact operator solution of the quantum Liouville field theory. Ann. Phys. 147, 365 (1983); Quantum Bäcklund transformation for the Liouville theory. Phys. Lett. B 118, 115 (1982)

5. Brézin, E., Douglas, M., Kazakov, V., Shenker, S.: The Ising model coupled to 2D gravity, a non-perturbative analysis. Phys. Lett. B 237, 43 (1990)

6. Brézin, E., Kazakov, V.: Exactly solvable field theories of closed strings. Phys. Lett. B 236, 144 (1990)

7. Crnkovic, C., Ginparg, P., Moore, G.: The Ising model, the Yang-Lee edge singularity and 2D quantum gravity. Yale-Harvard preprint YCTP-P20-89, HUTP 89/A058

8. Chodos, A., Thorn, C.: Nucl. Phys. B 72, 59 (1974)

9. Curtright, T., Thorn, C.: Conformally invariant quantization of the Liouville theory. Phys. Rev. Lett. 48, 1309 (1982)

10. David, K.: Mod. Phys. Lett. A 3, 1651 (1988)

11. Distler, J.: Princeton Preprint, PUPT-1161

12. Dijkgraaf, R., Verlinde, H., Verlinde, E.: Topological Strings in $d<1$. IAS-Princeton preprint, PUPT-1204, IASSNS-HEP-90/71

13. Distler, J., Hlousek, Z., Kawai, H.: Super Liouville theory as a 2D superconformal supergravity theory. Cornell Preprint, CLNS 88/871 
14. Distler, J., Kawai, H.: Conformal field theory and 2D quantum gravity. Cornell Preprint, CLNS $88 / 84$

15. Douglas, M.: Strings in less than one dimension and the generalized KdV hierarchies. Phys. Lett. B 238, 176 (1990)

16. Douglas, M., Shenker, S.: Strings in less than one dimension. Nucl. Phys. B 335, 635 (1990)

17. Feigin, B.L.: The semi-infinite homology of Kac-Moody and Virasoro Lie algebra. Russ. Math. Surv. 39, 155 (1984)

18. Feigin, B.L., Fuchs, D.B.: Representations of the Virasoro algebra. New York: Gordon and Breach 1989

19. Feigin, B.L., Fuchs, D.B.: Verma modules over the Virasoro algebra. Lecture Notes Math. vol. 1060, p. 230. Berlin, Heidelberg, New York: Springer 1982

20. Felder, G.: BRST Approach to minimal models. ETH-Honggerberg Preprint, Zürich (1988)

21. Frenkel, I.B., Garland, H., Zuckerman, G.J.: Semi-infinite cohomology and string theory. Proc. Natl. Acad. Sci. USA 83, 8442 (1986)

22. Gervais, J.-L., Neveu, A.: Novel triangle relation and absence of tachyon in Liouville string field theory. Nucl. Phys. B 238, 125 (1984)

23. Ginsparg, P., Goulian, M., Plesser, M.R., Zinn-Justin, J.: $(p, q)$ string actions. Harvard Preprint HUTP-90/A015

24. Gross, D., Migdal, A.: Nonperturbative two-dimensional quantum gravity. Phys. Rev. Lett. 64, 127 (1990)

25. Hilton, P.J., Stammbach, U.: A course in homological algebra. Berlin, Heidelberg, New York: Springer 1970

26. Horváth, Z.: Induced 2D supergravity as a theory of free fields. Phys. Lett. B 234, 30 (1990)

27. Horváth, Z., Palla, L., Vecsernyés, P.: BRST cohomology and 2D gravity. ITP Budapest No. 468 (1989)

28. Kac, V.G.: Contravariant form for infinite-dimensional Lie algebras and superalgebras. Lecture Notes Phys. vol. 94, p. 441. Berlin, Heidelberg, New York: Springer 1979

29. Kac, V.G., Kazhdan, D.A.: Structure of representations with highest weight of infinite dimensional Lie algebras. Adv. Math. 34, 97 (1979)

30. Knizhnik, V.G., Polyakov, A.M., Zamolodchikov, A.B.: Fractal structure of 2D quantum gravity. Mod. Phys. Lett A 3, (8), 819 (1988)

31. Lian, B.H.: Semi-infinite homology and 2D quantum gravity. PhD Thesis, Yale University, May 1991

32. Lian, B.H., Zuckerman, G.J.: BRST cohomology and highest weight vectors. I. Commun. Math. Phys. 135, 547 (1991)

33. Lian, B.H., Zuckerman, G.J.: New selection rules and physical states in 2D gravity; conformal gauge. Phys. Lett. B 254, (3, 4), 417 (1991)

34. Lian, B.H., Zuckerman, G.J.: 2D gravity with $c=1$ matter. Phys. Lett. B 266, 21-28 (1991)

35. Polchinski, J.: Remarks on Liouville field theory. Texas Preprint, UTTG-19-90

36. Polyakov, A.M.: Quantum geometry of Bosonic strings. Phys. Lett. B 103, 207 (1981)

37. Polyakov, A.M.: Quantum geometry of Fermionic strings. Phys. Lett. B 103, 211 (1981)

38. Polyakov, A.M.: Quantum gravity in two dimensions. Mod. Phys. Lett. A 11, 893 (1987)

39. Rocha, A.: Vacuum vector representations of the Virasoro algebra, in: Vertex operators in mathematics and physics. Berlin, Heidelberg, New York: Springer 1983

40. Rocha, A.: Representation theory of the Virasoro and super Virasoro algebras: irreducible characters, in: The 10th Johns Hopkins workshop on current problems in particle theory. Singapore: World Scientific 1986

41. Rocha, A., Wallach, N.: Highest weight modules over graded Lie algebras: resolutions, filtrations and character formulas. Trans. Math. Soc. 277, 133 (1983)

42. Seiberg, N.: Notes on quantum Liouville theory and quantum gravity. Rutgers Preprint, RU-90-29

43. Zuckerman, G.J.: Semi-infinite homology of the Virasoro algebra. Unpublished notes (1986) 
